\title{
Die tagebuchartigen Aufzeichnungen des pfälzischen Hofarztes Dr. Johannes Lange über seine Reise nach Granada im Jahre 1526.
}

Mitgeteilt und erläutert von ADOLF HASENCLEVER.

Auf den folgenden Blättern veröffentliche ich eine Reisebeschreibung aus dem 16. Jahrhundert, welche uns an der Hand tagebuchartiger Aufzeichnungen von Neumarkt in der Oberpfalz nach Heidelberg, von dort durch Lothringen, Frankreich und Spanien nach Granada an das Hoflager Kaiser Karls V. führt; nach nur 14 tägigem Aufenthalte in der ehemaligen Residenz der Maurenkönige wird die Rückreise angetreten, die zum Teil dieselbe Route einschlägt wie die Hinreise, stellenweise aber auch, besonders in Spanien, von dieser abweicht.

Der Verfasser dieses Berichtes ist der Leibarzt Pfalzgraf Friedrichs, des späteren Kurfürsten Friedrich II. von der Pfalz (1544-1556), Dr. Johannes Lange ${ }^{1}$ ) aus Löwenberg in Schlesien,

1) Ūber Joh. Lange, geb. 1485, gest. 21. Juni 1565 in Heidelberg, vgl. den Artikel von E. Gurlt in der Allg. deutschen Biogr. (1883), XVII, 637f., wo auch die einschlägige Literatur angegeben ist. - Einige Ergänzungen und Berichtigungen bietet Erler : Matrikel der Universitāt Lejpzig II, 454, 520, 524; III, 952, sowie Ed. Winckelmann: Urkundenbuch der Universitāt Heidelberg (Heidelberg 1886) II, S.100, Nr.906: 22. Novenber 1545, "Johannes Lange von Lemberg, der freien Kunst und beider artznei doktor, schreibt dem Kurf. (Friedrich II. von der Pfalz), daß er, seinem Wunsch gemäB, eine Reformation der Universitāt Heidelberg schriftlich verfaBt habe, und überreicht dieselbe zur eventuellen weiteren Verbesserung." - Nach Jak. Wille: die deutschen Pfälzer Handschriften der Universitātsbibliothek zu Heidelberg des 16. u. 17. Jahrh. befindet sich dort Cod. Pal. Germ. VIII, 34 ein Brief Dr. Langes an Kurfürst Friedrich III. von der Pfalz, d. d. Heidelberg 24. April 1564 úber die Krankheit des Pfalzgrafen, ebenso noch einige medizinische Rezepte Langes über die Kunst, das Leben zu verlāngem; vgl. ebenda Register v. Lange, Joh. 
als medizinischer Gelehrter eine sehr bekannte und geachtete Persōnlichkeit, insbesondere durch seine früher viel grelesenen und nachgeahmten epistolae medicinales, ${ }^{1}$ ) das erste derartige Werk in Deutschland.

Der Wert der hier veröffentlichten Reiseschilderung liegt fast durchaus auf kulturgeschichtlichem Gebiete; was wir Neues an historischen Notizen zur Zeitgeschichte erfahren, ist ganz geringfügig, zumal ein anderer Teilnehmer an dieser Reise, der bekannte Annalist Hubertus Thomas Leodius in seinem Werk über Kurfürst Friedrich II. von der Pfalz, ${ }^{2}$ ) dem Charakter seiner Biographie entsprechend, die Falurt seines Herrn nach Granada und die Erlebnisse während derselben in den historischen $\mathrm{Zu}$ sammenhang der Zeitgeschichte bereits eingereiht hat.

Hier sei gleich eine Frage kurz gestreift, welche insbesondere für die quellenkritische Bewertung von Leodius' Werk von Interesse ist: hat er bei der Redigierung seiner Biographie die Aufzeichnungen Langes, welche ihm bei seiner Stellung in der kurpfälzischen Kanzlei jederzeit leicht zugänglich waren, benutzt? Mit Entschiedenheit nach der einen oder anderen Richtung hin läßt diese Frage sich nicht beantworten; die Möglichkeit einer Benutzung liegt immerhin vor, besonders eine Vergleichung der Beschreibung von Granada bei unseren beiden Autoren macht die Annahme, daß Leodius von Lange abhängig ist, nicht unwahrscheinlich, freilich ebenso gut bleibt die Möglichkeit bestehen, daß beide, da die Gewährsmănner, von denen sie bei ihrer Unkenntnis mit der Landessprache über die spanischen Verhält-

1) Medicinalium Epistolarum miscellanea varia ac rara cum eruditione, tum rerum scitu dignissimarum explicatione referta: ut earum lectio non solum Medicinae, sed omnis etiam Naturalis historiae studiosis plurimum sit emolumenti allatura. D. Ioanne Langio Lembergio, Illustriss. Principum Palatinorum Rheni etc. Medico, autore. Basileae. Per Ioannem Oporinum. Ohne Jahr. Nach Gurlt in AD B erschien die erste Auflage in Base! 1554. Ich benutze das Exemplar der $\mathrm{Kgl}$. Bibliothek zu Berlin, wo auf dem Rücken des Einbandes $n$ Basil : 1554" eingedruckt ist. - Eine zweite, wesentlich vermehrte Ausgabe der epistolae medicinales erschien: „Francofurdi Apud Heredes Andreae Wecheli, Claudium Marnium et Ioann. Aubrium," 1589, herausgegeben von Nicolaus Reusnerus Iurisconsultus. "Cum Indice rerum et verborum copiosissimo." Vgl. über diese Ausgabe ADB a. a. O. Es wāre meines Erachtens eine sehr dankbare Aufgabe, die Weltanschauung dieses viel gereisten Arztes auf Grund seiner epistolae medicinales einmal des Nāhern zu skizzieren. Vgl. unten S. 433, Anm. 1.

2) nAnnalium de vita et rebus gestis Illustrissimi Principis Friderici Il Electoris Palatini Libri XIV, Authore Huberto Thoma Leodio, eiusdem Consiliario.“ Frankfurt a. M. 1624. - Fernerhin zitiert Leodius. - Die Reisebeschreibung des Leodius befindet sich Leodius a. a. O. S. 95-115. 
nisse und Kulturzustände unterrichtet wurden, die gleichen waren, auf deren Berichte hin, ein jeder für sich, ihre Aufzeichnungen gemacht und danach später ihre Erlebnisse und Erfahrungen ganz unabhängig voneinander niedergeschrieben haben.

Der Wert des hier veröffentlichten Tagebuches liegt, wie bereits erwāhnt, nach der kulturgeschichtlichen Seite hin; für die Flora der durchwanderten Lānder, für den Reichtum des Bodens an landwirtschaftlichen Erträgnissen hat unser Verfasser ein offenes Auge. Besonderes Interesse beanspruchen die zusammenfassenden kulturhistorischen Überblicke über die Kulturzustānde in den einzelnen Lāndern; man sieht, welchen Gefahren und Entbehrungen sich damals die Deutschen, auch Personen fürstlichen Standes, auszusetzen hatten, wenn sie ihren Kaiser in seinen fernen spanischen Erblanden aufsuchen wollten; gerade für die Geschichte des Reisens im 16. Jahrhundert, ein Kapitel, an welchem die amtlichen Relationen meistens stillschweigend oder doch, ohne sich auf Einzelheiten einzulassen, vorübergehen, enthālt unser Bericht manche schātzenswerte Notiz.

Dr. Langes Stellung zur religiösen Frage scheint wie diejenige seines Herrn, wie auch seines Reisebegleiters Leodius, keine bestimmt ausgeprägte gewesen zu sein; äuBerlich ist er noch ein Anhänger der alten Lehre, aber sein Auge ist bereits geschärft für die großen Gebrechen seiner Kirche. Nicht ohne Teilnahme verfolgt er die neue Richtung; charakteristisch ist in dieser Hinsicht seine Beurteilung Briçonnets, des Bischofs von Meaux, und des Vorgehens der Sorbonne gegen ihn.

Die Heimat des Verfassers ist Schlesien, die Gegend jedoch, an der sein Herz hängt, ist das kleine Ländchen seines Herrn, die Oberpfalz und die umliegenden Reichsstädte. Immer wieder, wenn er die GrōBe fremder Städte und Flecken erläutern will, greift er auf die geographischen Zustände dieses Lāndchens zurūck; die Ortschaften Amberg und Neumarkt sind für ihn die Maßbegriffe, nach denen er die Größe anderer Städte bestimmt; für bevölkertere Kommunen werden Nürnberg und manchmal auch Augsburg herangezogen.

Für den Statistiker sind diese Angaben ja kein geradezu 
ideales Material, nach dem sich genaue Berechnungen anstellen ließen; denn nicht nur wissen wir noch nicht genau, wie groß jene Flecken in der Oberpfalz damals waren, sondern noch mehr: was unser Verfasser angibt, sind immerhin nur Schätzungswerte, sie geben den Eindruck wicder, welchen der betreffende Ort bei dem meist nur ganz kurzen Aufenthalt auf den Reisenden gemacht hat.

Die eigenhändige Aufzeichnung Dr. Langes ist nicht mehr auf uns gekommen; die Handschrift, welcher diese Veröffentlichung entnommen ist, ${ }^{1}$ ) ist eine sauber geschriebene Kanzlistenhandschrift, niedergeschrieben, wie eine Notiz auf dem Titelblatt ergiebt, im Jahre 1528, wie ich annehmen möchte, entweder als Vorbereitung für eine Drucklegung - die Hervorhebung der Namen von Personen und Städten durch rote Buchstaben oder durclı mehr oder weniger willkürlich ausgeführte Einrahmung diescr Namen in rote und schwarze Kreise scheint mir darauf hinzuweisen -, oder die Handschrift war eine vielleicht für Pfalzgraf Friedrich veranstaltete Prachtausgabe. ${ }^{2}$ ) Soweit ich durch Anfragen und persönliche Nachforschungen ${ }^{8}$ ) habe ermitteln können, ist eine Verōffentlichung dieser Reisebeschreibung bisher nicht erfolgt, und sollte sie erfolgt sein, so sind die Exemplare des ersten Druckes heutzulage verschollen.

Da die Handschrift nicht Originalniederschrift des Verfassers ist, sondern von Kanzlistenhand herrührt, habe ich die überdies nicht einheitlich durchgeführte Orthographie - selbstverständlich nicht bei Städte- und Personennamen - der heute allgemein geltenden Editionspraxis von Urkunden aus jener Zeit angepaßt.

1) Aus einem Sammelband der Universitätsbibliothek zu Heidelberg; vgl. Jakob Wille : die deutschen pfãlzer Handschriften des 16. und 17. Jahrhunderts. Cod. Pal. Germ. 127; Pap. XVI. Jahrh., 330 Blätter [u. I-III leer] 2". I mit der alten Bezeichnung C 115 . Der Verwaltung der Universitātsbibliothek zu Heidelberg sei an dieser Stelle für die große Bereitwilligkeit, mit welcher sie mir die Benutzung der Handschrift durch Übersendung nach Halle ermōglichte, mein verbindlichster Dank ausgesprochen.

2) Gegen diese Aunahme kōnnte man allerdings einwenden, daß die Handschrift ohne irgend einen ersichtlichen Grund und ohne jede Schlußbemerkung ganz plötzlich abbricht, bevor die Reisenden den Ausgangspunkt ihrer Fahrt wieder erreicht hatten.

9) Bei R. Foulché-Delbose: Bibliographie des voyages en Espagne et en Portugal (Paris 1896) S. $26 \mathrm{ff}$. ist unter den Reisen Pfalzgraf Friedrichs diejenige von 1526 nur in der Schilderung des Hubertus Thomas Leodius erwāhnt. 


\title{
„Vfzaychnus des wegs mein gnediger her hertzog Friderich sambt seyner f. g. hofgesinde 1526. Jar in Hispania zwe Kayserlicher ma : $t$ zogen und wie es inen ergangen ist."
}

\author{
1528.
}

Got gibt got nimbt.

W. Sinderstetter.

Anno Tausent funffhundert und im sechsundzwentzigisten Jare ist der durchleuchtig hochgeborn Furst und herre, Herr hertzog Friderich Pfaltz[g]rave bey Rhein und hertzoge in Bayrn, unser gnediger herr, durch merckliche ursachen seiner furstlichen gnaden Landtschaff nutz und ander herren anligende beswerdnus betreffende verursacht, ${ }^{1}$ ) am dritten Tage des monats marcy mit disen hiernach geschriben seiner F. G. Räte und dienern zum Neuenmargkte, im Norgkau gelegen, gegen Granathen in Hisponiam disen verzaichetten wege durch Teutz-Nacion, Franckreich, Castanien, ?) Pasha, Pashaia, Castilien und ander Tayl Hisponier Landts zu kayserlicher Mayestat gerithen.

Friderich, Pfaltzgrave Bey Rhein, hertzog in Bairn.

Der wolgeborn herr, herr Georg von Falckenstein, frey und herr zu Haydeck, ${ }^{3}$ ) Rate und diener.

der ernvest Junckher Wolff von Mulheim, Marschalck.

1) Ūber Friedrichs Beweggründe zur Reise vgl. W. Friedensburg: Der Reichstag zu Speier 1526 (Berlin 1887) S. 117 ff., bes. S. 123 und 124, Anm. 1, sowie Rodriguez-Villa: El Emperador Carlos V y su corte (1522-1539), Madrid 1903-05, S. 327. 1. Er habe gehōrt, daß der Kaiser gesagt habe, er habe Maclit, die Pfalzgrafen zu bestrafen, und daB er nicht wisse, auf Grund welcher Tatsachen dies Karl gesagt habe. ,,S. M. respondió, que tal no habia dicho por ellos, pero bien era verdat haber dicho que era en su poder castigar a todos los que hiciesen porqué y fuesen deservidores." 2. Er wolle die Grōnde darlegen für seinen Rücktritt vom Reichsregiment. 3. Er habe den Kaiser und die ihm eben vermählte Kaiserin begrüBen wollen „y les dar la enhorabuena de su casamiento".

2) Gascogne.

3) Bei Leodius S. 96 a nur angeführt als „Dominus Georgius, Baro ab Heideck“" Im Türkenkrieg 1532 rar er einer der sechs Kriegsräte Pfalzgraf Friedrichs. 
der Ersam Hochgelert herr Johann Lange, $\left.{ }^{1}\right)$ doctor der Artzney von Lemberg.

der ernvest Jobst Brantner der Junger.

der ernvest Georg Brunbeck. ${ }^{2}$ )

Hans Bock.

Ruprecht von Luttich, Notarius. ${ }^{3}$ )

Gregorius Mayr, Silberschliesser.

Arnoldt Han, koch.

Jakob Lange, Lambarder.

Stephan, Sattelknecht.

Hans RagaB.

Paulus Kerner.

Hans von Amberg.

Joan. Laretha, ${ }^{4}$ ) Lacay.

Bastel, Bartwirer. ${ }^{6}$ )

Leonhart Fechter, kuchenbub.

Joan. Albertyn, Eseltreiber.

Vincens von Stockarth, stalknecht, $\left.{ }^{6}\right)$ und auch etzliche ander fremder Nacion knechte und diener, uff dem wege uffgenomen, und seiner F. G. zugeschickt. Und erstlich von dem obgemeiten Neuenmarckt gegen Norgkau

$$
\text { Berngrys }{ }^{7} \text { ) Meyl gezogen. }
$$

Ist ein Stetlein des Bischoffs von Eystett, under dem Schloß HirBperg an der Altnulul vischreich wasser gelegen, an welches wir nach essens mit meinem G. Herren gespacireth und darnach vor der herberge mit einem karn vol neuer Häffen Balspil geübeth.

1) Leodius S. 96a: "Doctor Ioannes Langius, Medicus tam eruditus quam suavis et iucundus comes". Auf S. 5 b nennt ihn Leodius: "Principis insignis medicus".

ग) War, wie aus Leodius S. $96 \mathrm{~b}$ hervorgeht, Mundschenk, ein wackerer Zecher. Vgl. Leodius S. 103 b.

7) Der Oeschichtschreiber und Biograph Pfalzgraf Friedrichs Hubertus Thomas Leodius. Vgl. hierzu Leodius: "et ego quoque ascitus sum Secretarius et a rationibus et sumptibus scriba" (a.a.O. S.96 a). Vgl. über ihn Hartfelder in den Forschungen zur deutschen Geschichte, Bd. XXV.

9) Wahrscheinlich identisch mit dem bei Friedensburg: Der Reichstag zu Speier 1526 3. 458, Anm. 3 erwāhnten Johann Marie.

") Leodius S. 112 a : „Principis tonsor Sebastianus".

o) Vgl. Leodius S. 96a: „Eramus autem onnes viginti"

7) Beilngries. - Wie auch aus Leodius S. 94 a hervorgeht, war die damalige Form des Namens Berngries. 
4 Meilen.

Ingolt-Statt.

Ein zirliche wolgepautte Stat der herrn von Bairn, uff einer ebent gelegen und kornreich Landt; die Donau daran hinfliesset und von der universthet auch berumbmet. Hatt auch ein woll erbauet SloB, in wellichem hertzogen Wilhelm ${ }^{1}$ ) und Ludwig $^{2}$ ) gebruder, Fursten und herren in Bairn, der hochwirdig in gott und durchleuch furst hertzog Philips, ${ }^{3}$ ) Pfaltzgrave bei Rhein, hertzog in Bayrn und Bischove zu Freysingen, auch hertzog Otto Heinrich ${ }^{4}$ ) und hertzog Philips ${ }^{5}$ ) gebruder, Pfaltzgraven bei Rhein und hertzogen in Obern und Nidern Bayrn, auch ein Junger Grave vom Algaw und ein herr von Bern meinem gnedigen herrn erhafftig entpfangen und zwen Tage allerlay kurtzweille gepflegt ${ }^{\circ}$ ) und sonderlich am Montag ?) nach essens Antvogel ${ }^{8}$ ) am Wasser gepaist; ${ }^{9}$ ) den andern tage darnach, uff das kein freude one laydt befunden wurde, ist mein gnediger herre von Freysingen am Schwengel kranck gelegen; und [es] hatt bede tag geregeth.

3 Meilen.

Neuburg an der Donau.

Ein Stat der jungen fursten und Pfaltzgraven uff ainem berge an der Donau gelegen, ${ }^{10}$ ) hat lustige jageth (ader gegaydt) und ein junckfrau Closter, in welchem die durchleuchtige furstin,

1) Wilhelm, Herzog von Bayern 1508-1550

2) Ludwig, Herzog von Bayern 1508-1544.

3) Bischof Philipp von Freising (1499-1541), Administrator und Bischof von Naumburg 1517-1541. Über den Leumund, in dem er bei seinen Zeitgenossell stand, vgl. Barack: Zimmerische Chronik IV', $187 \mathrm{f}$.

1) Pfalzgraf von Neuburg 1507-1556; Kurfürst von der Pfalz 1556- 1559.

5) Pfalzgraf 1507 - 1548. Beide Sōhne Pfalzgraf Ruprechts, Neffen Pfalzgraf Friedrichs.

6) Über einen politischen Auftrag an den Kaiser, den die bayrischen Herzoge durch Pfalzgraf Friedrich vortragen ließen, vgl. Riezler: Geschichte Baierns IV, 208.

7) 5. Mārz.

B) Zahme Entc; vgl. Grimm: Deutsches Wörterbuch. Leipzig 1854. I, 507.

9) gepaist $=$ gejagt.

10) Eire anschauliche Ansicht der Stadt und ihrer Umgebung, von Süden aus, aus dem Jahre 1546 ist dem 63. Jahrgang des Neuburger Kollektaneenblattes (Neuburg 1899) vorgedruckt. - Der Florentiner Serristori schildert die Lage der Stadt im September 1546 folgendermafen: „Nieumburgh ... è posto sul Dannubio, sito per natura assai gagliardo, sendo su un colle spiccato; et saria molto più, se non havessi un poggetto a cavaliere, è di forma rotonda, circundato per più della metà da fossi profondi et secchi, el restante bagna el fiume, ciuto di due muraglie per la maggior parte." (Friedensburg: Nuntiaturberichte J, Bd. 1 X, S. 597.) 
frau Margaretha, hertzog Georgen $\left.{ }^{1}\right)$ Seligen geborne tochter und ein Swester der obgemeiten Jungen Pfaltzgraven Muter, frauen Elbethen, Ebtissin ist, und aldo ist Anders Hiltner und Maister Benedict Stainschneider zu meinem G. Herrn kumen.

4 Meilen.

Wemdingen.

Nona Marcy.

Ist ein klain Stetlein, der herren von Bairn, do wir bey einer bosen unvertraglichen ehe-, sonder doch von einer holdtseligen wirthin seint berherbergt worden.

\section{Schwabenlande.}

3 Meil.

$$
\text { Sewingen. }
$$

X. Marcy.

Ein dorff in einem gantzen fruchtbaren und getraidreichen landt, nachent bey Norlingen gelegen.

2 Meil.

$$
\text { Elbang. }{ }^{2} \text { ) }
$$

Ain offen Stettlein mit sambt der Probstey hertzog Hainrichen ${ }^{8}$ ) Pfaltzgraven und Bischoff zu Utrich zustendig. Do ist drey schefflen gutter vische und der habern meinem gnedigen herrn geschenckt worden.

3 Meil.

$$
\text { Gayldorff. }
$$

XI. Marcy.

Ist ein klains stetlein, in grunde gelegen, Schenck Wilhelms, ${ }^{4}$ ) welcher meinem G. $H$. erhafftig beherberget, mit aller expens genugsam versorget. $\mathrm{DiB}$ Stetlein hat sonderlich von Natur wolgepiltte und schöne weybsbilder. Alldo ist herr Wolff Diettrich ${ }^{5}$ ) mit dreien pferden zu uns komen, und meinen G. H. paß gegen Ponth hinder Cuniagk beleyltet.

1) Georg der Reiche von Bayern-Landshut, geb. 1455, gest. 1503.

9) Ellwangen.

9) Heinrich, Bruder Pfalzgraf Friedrichs, geb. 1487, Bischof von Worms 1523-155', von Utrecht 1524-1528, von Freisingen 1541-1551; gest. 3. Januar 1552.

4) Vgl. über ihn Barack: Zimmerische Chronik III?, 62 ff. Er staminte aus dem reichsgräflichen Geschlecht der Schenken und Herren von Limpurg-Gaildorf, gest. 1552.

3) Wolf Dietrich von Knörringen. Er war ein Beamter Herzog Wilhelms von Bayern: 1527 finden wir ihn als Pfleger in Schwabeck (Chroniken der deutschen Stādte: Augsburg (1896) V, 244, Anm. 2), desgleichen 1539 (Roth: Augsburgs Reformationsgeschichte. 1904. II, 445). 1528 und 1532 wird er als Pfleger in Friedberg bei Augsburg erwähnt. $\mathrm{Ob}$ Wolf Dietrich einen politischen Auftrag an den franzōsischen König hatte, vermag ich nicht anzugeben; wahrscheinlich ist es. 
3 meil.

$$
\text { Öring am Kocher. }
$$

Ein zimliche statt der Graffen von Holoch, ') der auffrurischen Baurschafft auch anhengig geweBen. $\left.{ }^{2}\right)$

2 Meilen.

$$
\text { Wympfen. }
$$

Ein grosse Reichstat, vor Christi geburt Cornelia ${ }^{8}$ ) genant, hat einen Thumbstifft und leydt am anfang des Neckertals. Alldo ist mein G. herr mit dem von Haydeck, Wolff Ditterichen von Knerigen, Wolffen von Mulheim, Jobsten Prantner, Bastel Partbirn, Arnolden Koch uff dem Necker gegen Erberbach, meines g. herrn Stat, gefaren und die nacht aldo gelegen und an dem 14. tage Narcy gegen Haidelbergk gefaren.

\section{Der Neckertal.}

Ist ein gantz lustiger tall, in welchem uff beyden seythen dise nachvolgende sclılosser gebaut syndt. Erstlich Harneck Ernberg, darnach Horneck, ein schloß der Teutschen herrn, von den paurn außgebranth und zerrissen, darnach Hornberg, Gotzen von Berlingen, der paurn vor Wirtsburg veltfluchtigen hauptmans. Nachvolgent Ochausen, Bartholomey von Roß sloß, darnach Mynnenburg, Wilhalms von Haberns ${ }^{4}$ ) und ander slosser vil mer.

5 Meil.

$$
\text { Haidelberg. }
$$

Ist der Pfaltz ${ }^{5}$ ) Churfurstlicher sitz, am Necker zwuschen den bergen gelegen; hat ein Unniversithet und auff den berge ein groß wolerbauethes SloB mit selbentspringenden brunnen, welchs mein gnedigster herr Pfaltzgrave Ludwig) mit wall, schutten und thurmen und Mauren eflicher zwaintzig schue dick bevestiget; hatt an bayden bergen, am ende des Neckertals

1) Hohenlohe.

2) Über deu Verlauf des Bauernkrieges im Hohenloheschen vgl. Jak. Sturns Bericht rom 22. April 1525 bei Virck: Polit. Corr. v. Straßburg l, $196 \mathrm{f}$.

9) Vgl. zum Ursprung und historischen Wert dieser Legende Heid: Geschichte der Stadt Wimpfen, Darmstadt 1836, S. 19 ff., sowie A. von Lorent: Wimpfen am Neckar, Stuttgart 1870, S. 1 if.

1) Kurpfälzischer Marschall; seit 1524 .

5) Or.: der der. $\quad$ 6) Kurfürst ron der Pfalz 1503-1544. 
ligende, überflussigen fruchtbar weinbachs, Necker wein und Stroßberger genanth und uber den Rhein Pfedershemer, und im tall negste an der Stat zwen lustige welde, darauß allerlay wildts in eben felts mit sonderlicher lust und kurtzweil zu jagen ist. Aldo haben hertzog Hainrich Bischoff zu Utrich und hertzog Wolffgang ${ }^{1)}$ auch mein $G$. herrn entpfangen und seindt aldo die Osterlichen zeyt verharret. ${ }^{2}$ )

3 Meil.

Manaim.

Ist ein offen Stetlein, ain Meil uber den Rhein gelegen; aldo seindt bede obgemelten Pfaltzgraven die nacht bey meinem G. H. bliben, und am Rhein an der uberfurth leydt ein slo $B,{ }^{8}$ ) auf welchem der pfaltzgrave einen Bapst Scismaticum hat gefangen gehalden.

4 Meil.

\section{Neuestat.}

Ist ein Stat zwuschen fruchtbaren weinbergen am anefang des tals gelegen, und nahent uff einem berge an der Stat ist ein lustigs haus, Wintzingen genant, uff wellichem mein gnediger herr hertzog Friderich geborn ist. $\left.{ }^{4}\right)$

In diser Stat hatt der Bischoff von Speyer ${ }^{5}$ ) sich zu meinem $G$. herrn verfuget und im eerhe erzaiget. Ist ein alte Stat, in welcher kirchen des Pfaltzgraffen Rupprechts Romischen konigs her vater, ${ }^{\circ}$ ) der eyne konigin auß Arrogania ) gehabt hat, und Pfaltzgrave [Ludwig III.], s) der eine konigin auB Engelandt ge-

1) Der jüngste Bruder Pfalzgraf Friedrichs, ein Anhānger Luthers; vgl. über ihn Bossert in ZGO. XVII, 58, sovie R. Salzer: Beiträge zu einer Biographie Ottheinrichs, Heidelberg 1886, S. 24: ,Er hatte eine gelehrte Bildung empfangen und glich in seinem spāteren Leben und in seinen Neigungen am meisten Ottheinrich."

2) Über die politischen Verhandlungen rährend Friedrichs Heidelberger Aufenthalt vgl. Friedensburg: Der Reichstag zu Speier 1526, S. 124 ff. - Hier erst scheint sich Leodius dem Gefolge des Pfalzgrafen angeschlossen zu haben, wenigstens datiert erst von Heidelberg ab sein Reisebericht.

3) Die Burg Rheinhausen.

1) Am 9. Dezember 1483; vgl. Leodius S. 2 a.

5) Georg, seit 1513 Bischof von Speier, ein Bruder Pfalzgraf Friedrichs, geb. 10. Februar 1486, gest. 1519.

0) Ruprecht II., Kurfürst von der Pfalz (1390-1398).

j) Beatrix, Tochter des aragonischen Königs Peter II. von Sizilien; vgl. Häusser : Geschichte der rheillischen Pfalz I, 212.

9) Lücke im Text. Er war vermählt in erster Ehe mit Blanka von England, gest. 1409 
heurath hat, begraben sindt. Zwuschen Haydelberg und Neuenstat ist sechs meylen lang ein eben getraydreichs und vischreichs landt, mit uberflussiger weinwachs getziret, also bequeme gelegen, das man gegen Wurmbs, Speyr, Haydelberg in einem halben tage und eeher von einer Stat in die andern reythen oder geen mag.

\section{Kaiserslautern.}

6 Meil.

Quinta die Aprilis.

Ist ein wolgebauthe Reichstat, der Pfaltz versetzt ${ }_{1}{ }^{1}$ ) in wellichem[!] kayser Friderich Barba Rossa genant, uff den wellischen gebrauch und haydenische art, ein schlos hat angefangen $\mathrm{zu}$ bauen ${ }^{2}$ ) und nicht volendet; von der Neuenstat dahin zeucht man vier meylen zwuschen den bergen und wasser, in welchem foren und holtz gegen der Neuenstat fließen.

2 Meil.

$$
\text { Lantstal. }{ }^{3} \text { ) }
$$

Ein Slos des Frantzen von Sickingen gewest, in welchem $\mathrm{er}^{4}$ ) durch Pfaltzgraven Ludwigen und Bischove von Trier, ${ }^{5}$ ) bede Churfursten, und den Lantgraven von Hessen belegert. Ist durch ein SchieBloch o) yn Neuenbaue gestossen und in einem klaynen gewelbe ober dem weinkeller gestorben. Ist vast zerbrochen und mit dem umbgeschossen thurmb verfället.

\section{Köbelburg. ${ }^{7}$ )}

Ist ein dorff der Baurschafft das Reich genant, welliche die andern auffrurigen Bauren gefangen haben und bestricket; ${ }^{8}$ ) do sein wir die nacht gelegen und von den Baurn bewacht worden mit sambt unsern reysigen auch uffs veldt verordnet.

1) Endgültig seit dem Jahre 1417.

2) Im Jahre 1152.

3) Landstuhl.

1) Vgl. hierzu H. Ulmann: Franz von Sickingen S. $371 \mathrm{f}$.

5) Richard von Greiffenklau (1511-1531).

9) Vgl. die verschiedenen Angaben über den Ort und die Art der Verwundung bei Ulmann a. a. O. S. 371, Anm. 1, und S. 372, Anm. 2.

7) Kibelberg; vgl. zum dortigen Aufenthalt Leodius S. 96 a.

8) Bei Hartfelder : Zur Geschichte des Bauernkriegs in Südwestdeutschland (Stuttgart 1884) wird von dieser Episode, welche auch Leodius (S. 96a) ervāhnt, nichts berichtet. 
2 Meilen.

Santh-Wendel.

VII. Aprilis.

Ein ziemliche Stat des Bischoffs von Trier, wellicher aldo ist meinem $G$. herrn entgegen geritten und seinen Gnaden vil errhe erzaiget. In diser Stat wircket gott durch Sanct Wendel vil wunderzaichen und ist aldo leybtlich begraben, und sein $\mathrm{Er}$ hobner Corper uff den hohen Altar gestalt. Aldo ist einem Maurer ein stain mer dan hundert zentner swar uffs haubt, durch die gnade gottes an allen schaden, gefallen.

Aldo macht man Calcedainen Pater noster. Dise Stat hat Frantz von Sickingen dem Bischoff angewunnen ${ }^{1}$ ) und widerumb verloren. ${ }^{9}$ ) Aldo sein wir von dem Bischoff zwen Tage uffgehalden worden. ${ }^{3}$ )

5 Meilen.

$$
\text { Felschberg. }
$$

Auff dis wolgebauths lotiges ${ }^{4}$ ) Slos hat der Philips Helmstetter ${ }^{5}$ ) meinen gnedigen herrn geladen und mit funfftzig pferden wolbeherberget und vil eerhe erzaiget. Ein viertayl w'egs under dem Slos leyt ein Stetlein, ${ }^{\circ}$ ) do beraydt man die Lasur; aldo so wir uber das wasser, Moß genanth, gefaren seindt, ist zu uns komen der Grafen von Nassau ${ }^{7}$ ) und hat meinen G. Herren paß gegen Metz belaittet. ${ }^{8}$ )

6 Meilen.

Metz.

Ist ein wolerbauthe Reichsiat, alls groß alls funff Ambergk, hat mer dan sechzig kirchen und Closter und ein gasse, do man

1) An 3. September 1522 ; vg!. Ulmann a. a. O. S. $286 \mathrm{f}$.

9) Am 24. September 1522; igl. Ulmann a. a. O. S. 302.

s) Nach W. Friedensburg: Der Reichstag zu Speyer 1526, S. 124, handelte es sich allem Anschein nach um politische Aufträge an den Kaiser, welche Richard von Greiffenklau dem Pfalzgrafen mitzugeben hatte

4) Lotig=Gewicht habend, gewichtig; vgl. Grimm : Deutsches Wörterbucl.. Bd.VI. 1207.

5) Vgl. über ihn ZGO. XXIV, 39 ff., sorie ZOO. N. F. XVIII, 73 if.

6) Nach Leodius S. $96 \mathrm{~b}$ W'alderfingen.

ग) Graf Wilhelm von Nassau oder Graf Johann Ludxig von Nassau-Zureibrücken.

9) Wahrscheinlich hat sich Graf Wilhelm von Nassau, falls es sich hier um ihn handelt, zu Pfalzgraf Friedrich begeben, um die Schritte des Landgrafen in der katzenellenbogenschen Frage zu paralysieren ('gi. Meinardus: Der katzenellenbogensche Erbfolgestreit, Bd. I . Nr. 115). Dieses Schriftstück kann man (nach Friedensburg: Der Reichstag zu Speyer 1526, S. 124, Anm. 4) getrost auf dell 21. Dezember 1525 datieren. - Wie aus Meinardus a. a. O. S. 182 und 183 hervorgeht, stand Pfalzgraf Friedrich damals in dieser Streitsache mehr auf seiten Hessens; deshalb wird Graf Wilhelm wohl auch vermieder haben, ihm Briefe an seinen am Hoflager des Kaisers weilenden Bruder Heinrich mitzugeben 
uber die heuser reutthet und feret. Gibt dem kayser jerlichen tausent gulden tributs, welche sy nicht schuldig sein zu betzahlen, der kajser hole die dan in aigner person. ${ }^{1}$ ) Die Stat hat meinem $G$. herrn geschenckht und erhafftig belaytthet, hat umb sich einen fruchtbarn und mercklichen grossen weinwachs und funff meylen lang zu ritten, und wirt durch einen futh (oder vogth), von der Ritterschafft und Adel erwelt, geregirt. Dise statt hat Frantz von Sickingen im Weinlesen uberzogen und umb funffundzwaintzig tausent gulden geschatzet. $\left.{ }^{2}\right)$ Auch ist der stat Bischoff der Cardinal von Lottringen, ${ }^{\mathbf{s}}$ ) in welcher ein treffliche wolgebautte grosse kirchen mit vil umbgegen gebaut ist, darin ein Crucifix also groß alls ein khindt von zwayen Jaren hencket, man saget, es sey lauter golt.

\section{Meilen.}

Gorsia. $\left.{ }^{4}\right)$

duodecima Aprilis.

Ist ein offen margk und hat ein Abtey, dem Cardinal zu Lutringen zugehorig; uff disem wege anderthalbe meyle von Metz, als man uber das wasser Mosa genanth, welchs gegen stets (?) fleysset, [kommt], stet noch ein zerbrochener Aque ductus, ${ }^{5}$ ) von den Bolonesern genant Seratin, vor Christi gepurt gebauet, darauff das Trinckwasser in die stat Metz geflossen ist.

\section{Franckreich und Lotringen.}

\section{Aprilis. \\ Santh $\left.\mathrm{Mich} .^{\circ}\right)$ \\ 7 Meil.}

Ist ein klain stetlein an der Mosell ${ }^{7}$ ) und einem berge, daruff ain Closter ist gelegen; redet frantzosischs; uff diser tag-

1) Eine Notiz, die ich sonst nirgends belegt finde. Wahrscheinlich handelt es sich um die Renommisterei eines für die angeblicben Vorrechte seiner Vaterstadt begeisterten Lokalpatrioten. Gerade Kaiser Karl V. hat immer wieder trotz aller Remonstrationen seine Steuerkūnste an der freien Reichsstadt Metz mit großem Erfolg geübt.

2) Im Jahre 1518, nicht wāhrend der Fehde mit dem Erzbischof von Trier; vgl. zur Sache Westphal: Geschichte der Stadt Metz I, 339 ff., sowie Ulmann : Franz von Sickingen S. $97 \mathrm{ff}$., bes. S. 99, Anm. 2, wo die verschiedenen zeitgenössischen Angaben über die Höhe der Abfindungssumme verzeichnet sind.

₹) Bischof Johann, Herzog von Lothringen, aus dem Geschlecht der Guise (1505 bis 1550), seit 1518 Kardinal.

4) Gorze.

5) Vgl. über diesen Aquādukt Westphal: Geschichte der Stadt Metz. Metz 1875. Teil I, S. $16 \mathrm{f}$. - Noch heute sind Reste dieser rōmischen Wasserleitung bei Ars an der Mosel zu sehen.
6) St. Michiel
7) Unrichtig: an der Maas. 
rayse hat es in dreyen derffern gestorben, derhalben wir in einem futer ungessen 7 meylen geritten.

7 Meil.

Barleduck.

XIV. April.

Dis sind zwue Stet aneinander, dem herzoge von Lottringen von der Crone auß Franckreich gelyhen, in wellichen der konig von Franckreich $y m$ noch alle obrikheit behalten hat; die eine Stat mit sambt dem Slosse und thumb leut uff dem berge, die ander unden im tall an einem vischreichen lustign wasser; ist ein getraidreichs landt und hatt einen gantz mercklichen großen weinwachs uff den bergen und tâllern unubersichtlich.

\section{Schampania.}

7 Meil.

Vitrich. $\left.{ }^{1}\right)$

XV. Aprilis.

Ist die erst stath in Shampania, das ein kredigs Land, in wellichem [man] mit kreydenstein maureth; beherbergt vill kriegsleuthe und Buben, derhalben in einer meyle bey Utrich findet man siben galgen; die Stat ligt am Wasser Merla, ${ }^{2}$ ) welchs kayser Julius Matronam nenneth.

7 Meil.

\section{Schalon.}

XVI. Aprilis.

Leut auch in Schampania, ein Stat als groß alls Amberg; am Wasser; hat ein Bistomb und in einer klainen wolerbautten kirchen leut und ist Sannt Albinus begrebtnus und Sannt Lups heylthumb in einem Casten verschlossen; tregt man von einem dorff zum andern umb gelts wegen zu sameln. Aldo hatt mein G. H. Annillen und der herr von Haydeck und her Wolff Dittrich etzliche guldene Teffelein und ich Doctor Lange zway klaine ringle gekaufft von einem Pariser goltschmid.

\section{Ambry das landt.}

7 Meilen.

Pernes. ${ }^{3}$ )

XVIl. Aprilis.

Ist ein klaines und das letzte Stetlein Schampanie, hinder wellichem ain meile sich das Landt Bry und Ambry genanth

$\begin{array}{lll}\text { 1) Vitry-le-François. } & \text { 9) Marne. } & \text { 3) Epernay. }\end{array}$ 
anfanget; leydt zwuschen den bergen, doran holtz, wein und getrayde wechst und unden an den bergen wolgebautter vil dorffer, nicht ein kleine halbe meyll von einander gebauth; der tall hat ein grossen lustige wysenwachs (vil wiBmats), durch welches das wasser Merla, Latine Matrona, fleust, uff wellichem gegen Pariß holtz, kolen und weinpfele gefurt werden.

\section{Dorman.}

XVIII. Aprilis.

Ist ein klaines offen Stetlein am wasser la merla gelegen.

6 Meil.

Schettyo thyre. ${ }^{2}$ )

Ist Amberg in der grosse gemeB; am wasser und in einem berge gelegen, uff wellichem ist ein groß und weyths Sloß gebauth. Dise Stat mit sambt Dorman und andern zugehorenden dorffern, welche jerlicher Rendt zwaintzig tausent Crone einkomens haben, hat ko. nit einem gebornen Edelman deutzscher nacion, Ruprecht von Arnburgs Son, von wegen seiner ritterlichen that in veltschlachten geubeth seine Labtage langk gegeben, welchem man ist[!] von seinem schlos nennent Printz de Florania.

Item in disem tall von Schetthyottura bas gegen Alauerte muß man vier meyl uber das wasser Merla schiffen.

6 meil.

Alauerte.

XVIIII. Aprilis.

Ist ein dorff, darin man gutte herberg uberkommt.

\section{Me o us. ${ }^{2}$ )}

Ist ein alte Stat wol erbauet, Lateinischs Meldum genant, anderhalb Nurmberg gemeB, darvon das eussere tayl vom wasser Merla gantz absunder und umbflossen ist, derhalben nie das gewunnen noch irem herren abgefallen ist, darumb auch alles tributs befreyet. Hat einen freyen platz, daruff in einer kriegsordnunge funffundzwaintzig tausent man sten konnen, und auch ein Stifft, welchs Bischoff $^{3}$ ) von wegen der Lutherischen leer man zu Pariß hatt wollen verbrennen, und ist durch des koniges

1) Château Thierry. 2) Meaux.

7) Guillaume Briçonnet; vgl. Soldan: Geschichte des Protestantismus in Frankreich, Leiptig 1855, 1, 88 ff., sowie bes. Erich Marcks: Gaspard von Coligny, Stuttgart $1892,1,276 \mathrm{f}$. 
swester ${ }^{1}$ ) gonst doch bey dem Episcopath noch erhalden. In diser Stat sein sonderlich vil Iuchmacher.

7 Meil.

Selibri.

XX. Aprilis.

Ist ein dorff, drei meylen von Pariß gelegen.

3 Meil.

Pariß.

XXI. Aprilis.

Dise Stat haben wir mit sambt unserm gnedigen herren von einem hohen thurm besichtiget und funff Nurmberg gleichmessig geschatzet. Hat ein furtreffliche Universteth, welche kain kayserlich recht lernet, ${ }^{2}$ ) und die doch das Perlament gebraucht, und der Theologen halben auch mercklich abnympt. Am Montag vor essens hat das Perlament in pallast unsern gnedigen lierrn erhafftig entpfangen und unter ine erliche stelle gegeben; aldo haben wir zwue stunde ernstliche richtshandlunge und rechtlichen gebrauche gehört, auch hat man meinern gnedigen hern obgemeltes pallast alle Camern und gefencknus getzaiget, welche mit ubergultten tafelbergk und decken, auch seyden tapissrien wolgeziret syndt. Durch dise Stat flyssen geweltige wasser, uber welche ein klayne brucke, genanth der goltschmid, gebeuche hat bey hundert gleuchformige heuser; ${ }^{\mathbf{}}$ ) die ander große brucke hat vast zwayhundert gleichgebautter gutter kauffmansheuser; die stat enge und gepflasterte, stetig unfletige nasse wege und gassen; aldo habe wir zwen tage gerueth.

7 meil.

Montheri.

Ist ein offen Stetlein, hat auff dem berge daran gelegen ein Sloß und gutte weinwachs und getraidlandt, dohin der weg von Pariß mer dan halb gepflastert ist.

1) Margarethe von Navarra.

2) Vgl. La Orande Encyclopédie XXV, 568: „L'enseignement du droit et particulier du droit romain, interdit à Paris, y (in Orleans) fut surtout prospère", sowie R. Dareste: François Hotman (Rev. hist. 1. Jahrg., 1876) II, 2 ff.: "L'université de Paris n'enseigna que le droit canonique. Orléans, au contraire, n'avait qu'une faculté de droit civil, mais ancienne et illustre."

9) Vgl. L. Pastor: Die Reise des Kardinals Luigi d'Aragon etc., Freiburg i. Br. 1905, S. 131: "Tra quali ponti quello di li aurefici credo sia longo appresso cento passi, dove se lavora d'oro et d'argento tanto et cosi artificiosamente, come in parte del mundo.“ 
Die tagebuchartigen Aufzeichnungen des Dr. Johannes Lange. 401

\section{Das Landt Beaous. ${ }^{1}$ )}

7 Meil.

Eth a m ps.

XXV. April.

Ist ein stat fast als Paris ader ein virteil meylwegs lang, nicht uber zwayer gassen dicke, hat der konig seinem Camerlingen sein lebetage geschenckt und eingeben. Leudt im landt BeaouB genanth, welches sich baß gegen Orliens erstrecket, und ist nicht uber 3 meyle prayt; von getraide ser ein fruchtbar und eben landt, hat wenig weinwachs und noch weniger holtz.

10 meil.

Turi. ${ }^{2}$ )

Ist ein zimlich dorff, do wir der wirthin umb die kamerschlussel haben ducaten und Cronen und Stiffeln mussen verpfenden.

10 meil.

$$
\text { Orliens. }
$$

Ist ein Stat also groß alls Augspurgk, darvon auch das hertzogthomb, des konigs menlichen erben zugehorig, genanth wirt. Bauet von dem weintzehnet ein veste streubpere und zirhafftige maur, hatt einen vast fruchtbaren weinboden, darauff sonderlicher gesunther und schmackhafftiger clarer rotter wein wechst, hat auch in kayserlichen rechten ein berumbtte universithet und auch ein Bistumb; neben diser Stat fleist ein schiffreich wasser, Lateinischs Liguris genanth; und einen stain wegk von Thuri 8 meyle langk.

4 meil. Noster Damma d'Cleri. ${ }^{3}$ )

Ist ein offen marckt, do gott durch die junckfrauen Marie wunderzaichen wircket und der gottesdinst mit der briester nutz mit wachs prennen und auffgesteckten liechten vast geubt wirt, welche, so sie auffgesteckt sein, balde durch einen verordenten diener werden auBgelescht und nachvolgents wider verneuert durch die weyber vayl getragen und frembden leuthen eingezwungen zu kauffen.

In diser kirchen leudt konig Ludwig ') begraben. Dieser wegk ist auch uber das halbe tayl gepflastert, darbey auch fleist

1) Beauce, Landschaft im Sūdwesten von Paris, sehr getreidereich.

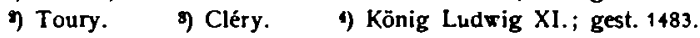

Archiv für Kulturgeschichte. $V$. 
das Wasser Semleyn genant, welches zwue meylen hinder Orliens auß einem grundlosen brunnen entspringt. Aldo hat Cunradt, Thumbher zu Bles und zu Thurs, des weyermaysters Son vom Neuenmarck purtig, meinem gnedigen herrn den Wein geschenckt und mit vier pferden belayth.

\section{3 [meil.] Santh Lorents. ${ }^{1}$ )}

Ist ein dorff, sauber lustige herbergen; uff disem wege hat obgemeltter herr Cunradt mein Gnedigen herren zu wolgefalle mith drey ploefussen ${ }^{2}$ ) alastern gepeyst; und auff der rechten handt lassen ligen zwue stette des Bischoffs von Orliens Beaucfi ${ }^{3}$ ) und Mōhe, ${ }^{4}$ ) und uber eine Meyle darnach ein stetlein Longa villa genant des Marggrafen von Rottelle, der vor Pavia erschossen ist; under sannt Lorentz fleust ein wasser yena genant, hinder welchem leyd ein thiergartten. DiB alles ist ein eben weinreichs lustiges Landt.

8 meil.

$$
\text { Bles. } \left.{ }^{5}\right)
$$

XXX. April.

Ist ein Stat Augspurgk in der grösse gleich an einem berge uber dem wasser Liguris genant gelegen, uff welchen leydt ein vest wolgebauts und zirhafftiges schlos, welches unden an dem Berge hat ubereander vier undergeschieden gerten ${ }^{8}$ ) mit $\mathrm{C}$ pressenpaume und granaten, opffel, maulpeerbaumen und weinhotten und andern edeln gekreuttern und prunnen wolgetziret, und sunderlich ym obersten garthen ist ein lustign kunstreicher Laborinth mit einem Summerheyßlein gemacht; auß disem Garten ist in das Slos ein eingangk, daran uff der lincken hannt ein hindtcontrafeth gestelt ist, ${ }^{7}$ ) weiches uff seinem haubte ein recht naturlich hirschsgehurne hat von XXII enden, welches Marggrave Christoff von Baden hat an einem hinde befunden und das dem

1) St. Laurent des Eaux.

2) ploefuessen: Blaufüsse $=$ Wanderfalken. Vgl Archiv für Kulturgesch. II, 1!ff.

$\begin{array}{lll}\text { 3) Beaugency. } & \text { 4) Meung. } & \text { b) Blois. }\end{array}$

b) Auch in der Zimmerischen Chronik (ed. Barack IIl2, 243) werden die schönen Gärten von Blois rühmend hervorgehoben. „In der stat bliben sie [die Grafen Zimmern] ain tag oder zwen, die stat, das schJoß und dann die schōnen Gärten zu besehen, wie auch geschach" "; ebenso bei L. Pastor a. a. O. S. 144.

7) Vgl. L. Pastor a. a. O. S. 144: „Intrato la porta del zardino ad man dextra è contrafacta una cerva con uno paro de corna grandi de una vera cerva, quale secondo diceva la inscriptione fu ammazata dal marchese di Bau, et la donò al duca del Rheno [Herzog René von Anjoul, et quello al roy Ludovico." 
konige von Franckreich zugeschickt mit versigelten getzeugknus etlicher edelleut, die das obgemelt wildt haben gesehen und helffen fangen, welcher namen auff einer taffel angeizaichnet under dem hindt hangen.

In disem garthen hab ich auch mit doctor Wilhelm Copo, $\left.{ }^{1}\right)$ des kuniges muter $^{2}$ ) leibartzet, kuntschafft gemacht.

In diesem obgemeltten Sloß [liegt] in einem verpichten Sarck des koniges von Franckreich eeliche gemahels ${ }^{8}$ ) Corper, vor zwayen Jaren verschieden, noch unbegraben von wegen der uncost, nemlich tausent Cronen, dy Irer begrebnus gebracht erfordert, und kriegs halben unbegraben, und wirth allererste im September dises Jars begraben werden.

Diß ist ein lustiger wecksteich neben dem wasser Liguris uff der rechtten handt fliessende, do zeucht man 6 meyl zwuschen seer fruchtbaren und wolgepauten weingertten und darnach 3 meyle auff einem eben getraidreichen Lande.

10 Meilen.

Ambas. ${ }^{4}$ )

Ist ein stat am wasser gelegen, dorin des koniges slos auff einem fels gebauth ist, ${ }^{5}$ ) in welches graben seinth drey grosse alde leben (?), und in disem sloB ein grosser schneck, ${ }^{\circ}$ ) in welchem man auff und abe reyten und faren kan.

\section{Das Land Thyrenia.?)}

8 meil.

Mantellan. ${ }^{8}$ )

Ist ein dorff im landt Thurenia, welchs dem Bisthumb Thurs zustendig ist, gelegen; auff disen acht Meylen ist mer getraidtswachs den weinwachs.

1) Der berūhmte Leibarzt König Franz' I., aus Basel gebūrtig; gest. 1532. - Sein Sohn Nicolas war bekann tlich befreundet mit Calvin.

†) Luise von Savoyen, geb. 1476, gest. 1531.

8) Claude de France, Tochter König Ludwigs XII., geb. 1499, vermāhlt 1514, gest. 1524.

4) Amboise.

5) Man vgl. Pastor: A. de Beatis Reisebeschr. S. 142 f.: „.... Amboys ..., quale si bene è poca villa, è allegra et ben posta; lei è in piano, ma ha un castello in pogecto, che si non è di fortezza è commodo de stantie et ha bellissima prospectiva."

B) In übertragener Bedeutung Wendeltreppe; hier wahrscheinlich Wandelgang.

$\begin{array}{lll}\text { 7) Touraine. } & \text { Manthelan. }\end{array}$ 


\section{Meil. Schatelria. ${ }^{1}$ )}

Ist ein Stat Amberg gleuchmeyssig des hertzogs von Burbon, $\left.{ }^{2}\right)$ von welcher Stat wege, so des konigs muter mit recht y'm angewonnen hat, ist obgemelter hertzog zum kayser gefallen.

Uff der virde meylen bey dem dorff Parthpiel ${ }^{3}$ ) genant seint wir uber das wasser kreude ${ }^{4}$ ) geschifft, welches man lateynischs Sycorym ${ }^{5}$ ) nennet, und uff der rechtten hant von Schatelria fleusset auch ein mercklich groß wasser, Wyenna genant; bey disem wasser hat Julius Cesar die Franzosen geschlagen. ${ }^{\circ}$ )

Auff disen 7 meylen eben landts weckhs[t] wenig weins und uberflussigk vill guttes getraidts, das pillich des Franckreichs kornhauB soll genant werden. Bey obgemeltem wasser kreuda endet sich Thurenia und fenget an das Landt Poytirs, Latheinischs Pittavia genant.

\section{Das Landt Pictavia. ')}

7 meyl.

Poytyrs.

Ist ein Stat grosser dan Nurmbergk und an der Lenge Pariß gleichmessig auf einem perge gelegen, in welcher ist ein Bisthumb und in der thumbkirchen leydt Sanctus Hilarius ein Bischoff begraben. In diser Stat haben wir erstlich das woisser mussen kauffen, sonder ${ }^{8}$ ) der wein ist von den Thumbherren und einem Rathe doselben meinem gnedigen herren geschenckt worden.

3 [Meilen].

\section{Vivon.}

Ist ein klaines Stetlein, do man auch hintzu der Mutter gottes und wol pillich gott zuvoran wallet, wan sie ye der gnaden und Barmhertzigkhait ist und vil genad zu erberben hat alls die muter gottes.

1) Châtellerault.

2) Karl von Bourbon, der Verràter, geb. 1490, geht 1523 auf die Seite Karls V. über, stirbt 6. Mai 1527 bei der Erstūrmung Roms.

9) Le Port de Piles. 4) Creuse, im Altertum Crosa genannt.

5) Es muB hier eine Verwechseiung oder Wortserstümmelung vorliegen; die Creuse heiBt auf latcinisch Crosa.

5) In dieser Gegend hat keine Schlacht zwischen Julius Cäsar und den Galliern stattgefunden. Wahrscheinlich wurde durch Lokallegende die Erinnerung an irgend eine frühere Schlacht mit dem berūhmten Römer in Verbindung gebracht.

7) Poitou. 8) $=$ aber. 


\section{Meil. Lusimer. ${ }^{1}$ )}

Ist ein kleines, von selbentspringenden brunen gerten und holtzwachs ein lustiges stetlein auff einem berge gelegen, do auch der hednische gothin Melusyn, von welcher die pfaltzgraven sollen ursprung haben, wolgebauts Schlos stet, in welchem der Burgundische hertzog von Urania gefangen gelegen hat; under dem schloß ym tall am berge, do ist der Melusyn brun, darin sie sich gebadet hat, und daruber ist ein neues kirchlein gebauet.

Daran unden im tall fanget sich an der thiergartten, ein deutsche meyle lanck, in welchem wir vierhundert stuck wilts gesehen haben, und durch disen garthen fleysset zwue meyl ein vischsreichs wasser. ${ }^{2}$ )

Man sagt, das obgemelte Melusina noch vor des Franckreichischen koniges und konigin tod zwen tage sichtigklich erscheyne. ${ }^{3}$ )

3 meil.

$$
\text { G o y. } \left.{ }^{4}\right)
$$

Ist ein kleines dorff, darin seint wir ein nacht gelegen.

7 meilen.

Bufetts. $\left.{ }^{6}\right)$

Ist auch ein klainer fleck, darynn wir auch seinth ain nacht gelegen.

3 meilen.

$$
\text { Mala. }{ }^{\circ}
$$

Leydt an einem grossen wasser. ${ }^{7}$ )

\section{Hertzogthumb Anguleim.}

4 meyl.

Angul ema.

Ist die haupthstat des obgemeltten hertzoghumbs, jetz des konigs mutter zustendig, auß weichem diser konig Franciscus geboren ist; ${ }^{8}$ ) hat ein zirlich Schlos mit einem lustigen garten

1) Lusignan. 2) Vonne.

3) $\mathrm{Vgl}$. Zimmerische Chronik II12, 49: ,Zu Lusingen, sagt man, wann ein künig von Frankreich sterben, so höre man etliche nächt darvor ein grausams geschrai umb das schloß, und das soll die Melusina sein."
4) Couhé
5) Buffec.
B) Mansle.
7) Charente.

B) Am 12. September 1494 in Cognac. 
und ein altte thumbkirchen, welche balde nach dem tode Sanct Petri ist gebautet worden. ${ }^{1}$ )

3 meyl. Schetgenau. $\stackrel{\text { ) }}{ }$

Ist ein kleiner offner margk, darinne wir seint ain nacht gelegen.

2 meylen.

Schyma u. ${ }^{3}$ )

Ist ein klains Stetlein an einem lustigen wasser gelegen, daran wir vast uber ein zerrissne unebne lange brucken haben mussen reytten und uberfaren. Darinne hat der Ammiral ${ }^{4}$ ) ein lustiges wolgebautes schloB mit lustigen sälen und Camern, welche mit sonderlicher wolgemachten tapissreien und bethen wolgeziret sein, darinne hatt $\mathrm{Er}$ meinen $\mathrm{G}$. herren beherberget und vil erhe ertzaiget.

2 meil.

$$
\text { Cuniagk. }{ }^{8} \text { ) }
$$

Ist ein Stetlein nit grosser dan der Neuenmarckt, in welchem der konigk auff diß mall hoff hielt. Aldo seint meineni G. herren zwin hertzogen von Lotringen, Musignor de Gol ${ }^{8}$ ) und sein Bruder von Vadmon, ${ }^{7}$ ) und der viceregh von Neapolis ${ }^{5}$ ) [entgegengeritten] und haben meinen gnedigen herrn in des koniges schlo $\beta$ belayttet und in des koniges Camer; ${ }^{9}$ ) aldo hatt der konig meinen gnedigen herren freuntlich mit freuden entpfangen und nachvolgens auch in seiner muter Camer und in das frauenzimer belayt und dan in seine gemach, welche sunderlich fur meinen genedigen herren verordent und beraydt worden. Dise obgemeltten Camern sein getziret gewest mit gulden und auch silberen tapisereien und etliche mit sameten

1) La Cathédrale St. Pierre, im XII. Jahrhundert erbaut, spāter restauriert.

2) Château neuf sur Charente. 9) Jarnac (?).

9) Philippe Chabot, seigneur de Brion, seit 1526 in dieser Würde; gest. $15+3$.

s) Cognac. Đ) Claude, premier duc de Guise (1496-1550).

7) Louis, duc de Guise, comte de Vaudémont, gest. 1528 vor Neapel.

8) Karl vor Lannoy. Über den Zweck seines Aufenthaltes am franzōsischen Hof und seine dortigen Verhandlungen vgl. Fr. Decrue: Anne de Montmorency, Paris 1885, S. 84.

9) Über des Pfalzgrafen Empfang vgl. Diarii di Marino Sanuto, Bd XLI, Sp. 384 (Bericht des Sekretärs Rosso vom 10. Mai 1526): ,è zonto qui il conte Palatino con 15 cavalli, va in Spagna. Lì andò contra monsignor di Lutrech, et c̀ stà honorato assai, alozò in castello col Vicerè. Li andò etiam contra monsignor il Gran Maestro et poi il Vicerè.." 
tapisreien, auff welchem die fabeln und geticht Virgily in Buccolicis mit golt und perlen kunstreich gestickt, und bethe wolgetziret.

Der konig hatt vierhundert harschirer, welcher jetzlicher zway pferd helt und hundert schweitzer, welche alle sambt von dem konige geclaidet werden und helleparthen tragen und tag und nacht uff den konig warten; disem koniglichen hoffe zihen kromer und kromer (sic!) und allerley kauffleute und hanckwergks Leutt nach, das man sechtzig tausent person, das mayste tayl berietten, schatzet dem hove nachziehen.

Item der konig hatt meinem gnedigen herrn obents und morgens ein freyhe furstliche taffel gehalden und alle ritterliche kurtzweil und lust mit meinem Gnedigen herrn gefleget, wie dan hernach volget.

Erstlichs Am Samstage ${ }^{1}$ ) nach essens haben sye mit sonderlichen woll abgerichtten hunden weiß und rattfarben unangekuppelt einen hirschen gejaget, uff welchen ungefordert kayner fur den Jeger lauffet, so sie doch gleuch das wilt sehen ader an Jagen horen.

Auff den Sontag ${ }^{2}$ ) nach essens haben sye sich in tuchern vorhaltten in gegenburt des frauenzimers gejaget und etliche Frischlinge gefangen und acht gestochen, under welchen ein weyBgescheckts befunden ist.

Auff den Abent hat der konig meinem gnedigen herrn ain Panckhett gehalden und aldo meinen gnedigen herren uber sich und den vicerege von Neapolis und Engelischen legat ${ }^{3}$ ) und Cardinal gesetzet, und nach essens einen tantz und Mummerey gehalden mit seyden und samathen kleydern verklaydet, darunder der konig und mein gnediger herre und der Cardinal und hertzog von Lotteringen erschinen und auch vercleydet worden.")

Auff den montag ${ }^{5}$ ) hat der konig meinen gnedigen herren $\mathrm{zu}$ tische in den garten geladen und auff den obent des konigs

$\begin{array}{lll}\text { 1) 12. Mai. } & \text { 2) 13. Mai. } & \text { ग) Thomas Cheyne. }\end{array}$

4) Daß neben diesen mannigfachen Vergnūgungen auch Zeit zu ernsten politischen Gesprāchen übrig blieb, geht aus Leodius' Darstellung $S$. $98 \mathrm{f}$. hervor. Über unmittelbare Auftrāge des franzōsischen Kabinetts an Pfalzgraf Friedrich für Kaiser Karl vgl. Fr. Decrue: Anne de Montmorency, Paris 1885, S. 84, auch Anm. 3

5) 14. Mai. 
muter in das frauenzimer. Item alle tage und obent seint des koniges Musici on meines Gnedigen herren tapfel und schloßkamer erschinen und freude gemacht.

$$
\text { Ponth. } \left.{ }^{1}\right)
$$

Ist ein stetlein alls der Neuenmarckt, hatt doch ain $\ldots{ }^{2}$ ) graben aller voll lustiges reynes flyssenden wassers. Ist einer witfrauen, welche meinen gnedigen herren geladen hat und freuntlich mit dem weyn und andern vereret.

Aldo ist herr Wolff Dietterich von Knorigen mit sambt seinem bruder und dem Srethebach von uns abgeschaiden.

Auch der konig meinem gnedigen herren einen reysigen botten und einen Edelman zugeordet, welche allen stetlein schrifftlichen bephel uberantwurt haben, daz sye meinem gnedigen herren alle erhe ertzaigen und nicht anders halden sollen, dan wers der konig in aigner person, welchs dan dem fleissig nachkomen und gescheen ist von allen nochvolgenden Stetlein.

4 Meil.

Etholie.

XVII. May.

Ist ein dorff, darin man gutte herberge und alle notturfft fyndet.

3 meil.

Blay.

Ist ein klains stetlein auff einem berge am eusern Merhe gelegen, in welcher vorstat ist ein alte gebautte kirche, do in der understen grufft auff der lincken handt leut Sanct Rolandt und uff der rechtten hant Sanct Oliveri, Santh Romanus Eucharius und Faustina und auff der lincken handt der staffel sich[t] man sannt Apolanie begrebnus. ${ }^{8}$ ) Aldo sein wir auff einem Arm des Mers siben meylen gegen Burdeos gefaren, pferdt und allen droB uffs schiff geladen.

1) Pons. 2) Ein nicht zu entzifferndes Wort; ich lese: ,druiachtigen".

3) Man vgl. damit Leo von Rozmital: Reise durch die Abendlande in den Jahren 1465, 1466 und 1467, beschrieben durch Gabriel Tetzel von Nümberg, herausgegeben von J. A. Schmeller in der Bibliothek d. literar. Vereins in Stuttgart, Stuttgart 1844, VII, 165: „Von dannen ritt wir auss etwan vil tagreis in ein grosse stat, heisst Plaa, do leit die heilige junkfraw sand Appolonia und sant, Rewerin'. Item do leit auch Olyfemus und der groß Rulant und sein schwester. Seind außdermassen groß leut gewesen. Des Rulant sch vester ist meiner spannen zweinzig lang gewest, und ir bruder gar vil länger und grösser gewesen." Zu diesen Mitteilungen vgl. man die angeblich auf Autopsie beruhenden kritischen Bemerkungen bei Leodius $\mathrm{S}$. 5 . 
Die tagebuchartigen Aufzeichnungen des Dr. Johannes Lange. 409

7 meil.

Burdeos. ${ }^{1}$ )

XVIII. May.

Ist ein kostliche altte stat, ${ }^{2}$ ) bey wellicher in ayner meylen lang in das merch flyessen treffenlicher grosser drey wasser: Dordonea, Gyrunda, Garunna. ${ }^{3}$ ) In diser Stat fyndet man noch vil altter haydenischer gebeue, nemlich templum Lutele und vor der Stat ein zerbrochen Theatrum; ${ }^{4}$ ) hat einen großen weinbachs uff der andern seythe.

v meyl. Budensa. ${ }^{5}$ XX. May.

Ist ein zimlich dorff, dohin wir auff dem wasser Garuna gefaren seinth, an welchs uber vill merhhunde sich samethen.

5 meil.

Longon. ${ }^{\circ}$

Ist ein klaines Stetlein, lateinischs Lyngonia genanth, neben welchem wir seint vorgerytten und die Burger und [Ambtleut] ') man nach koniglichem bevelch haben wein und Collacion uns auff den wege mit gedachter tafel angericht. Alhie fangen sich wider an grosse meylen.

\section{Castanier-Land. ${ }^{8}$ )}

Besas. ${ }^{9}$ )

Ist eine zimliche stat, Phasacum vor alders (oder vor zeitten) genandt; hat einen Bischoff und aldo haben sie Sannt Johanns pluet. Alhie ist der anfanck Castanier Landts, welches vast fruchtbar ist umb die obgemeltte Stat; sunder nachvolgens ist dreyssig grosser deutscher meylen ein gantz eben und sandichs unfruchtbars Landt, hat vill poser puben und kriegsleute, wenig wein und getraids und auch durre wayde.

1) Bordeaux.

9) Vgl. L. v. Rozmilal a. a. O. S. 165: „Von der stat auss (Blay) muosten wir mit unsem pierden über ein groß wasser varen, siben teutsch meil lang, in ein stat, heißt Burdeus, ist ser ein schone kostliche stat."

3) In Wahrheit ist die Gironde bekanntlich kein besonderer Strom, sondern das Ästuarium der vereinigten Flūsse Garonne und Dordogne.

4) Les ruines des Arènes, dites le palais Gallien.

$\begin{array}{llll}\text { 5) Podensac. } & \text { o) Langon. } & \text { 7) Durchstrichen. Bascogne. } & \text { 8) Bazas. }\end{array}$ 
3 meil.

Capsious. ")

XXI. May.

Ist ein kleines Stetlein, do man vil Eysenertzt vindet und die Statmauer auch darvon gemacht ist; hat schones viech und kynder.

+ meil.

Rockeforth.

Ist ein kleins Stetlein.

3 meil.

Monthmarschans. $\left.{ }^{2}\right)$

Ist ein Stetlein, darinne sich anfecht wunderbarliche schlayrung und klaydung der weyber.

+ meil.

Tartas.

Ist ein kleins Stetlein an einem grossen wasser gelegen, hat auch ein sloB auff dem berge, erkennet fur iren herren den vertriben konig von Navarr, ${ }^{8}$ ) hat auch ain zimlichen grossen Weinbachs. In diser Stat an dem pfynstmonedt ${ }^{4}$ ) haben sy einen Bischoff geklaydet und frauen und gesellen die nacht und gantzen tag mit sambt den pfaffen getantzet.

4 meil.

$$
\text { Ad Axs. } \left.{ }^{5}\right)
$$

Ist ein Stat Amberg gleichmessig an einem schiffreichen wasser, Dosa ${ }^{6}$ ) genant, gelegen, darin der konig von Franckreich ein groß tayll seines geschutz haldet. In diser Statt entspringt ein lautter clares warmpaedt, in wellichem man homer pruet und ayer syden mag, und von seiner hitz wegen muB an einem andern orthe zum bade gekuelet werden.

In diser Stat am pfingst eristag () haben sie eill groß schiff in trucker stat umbgetzogen und auff freyer gasse Collacion gehalden die schiffleute.

3 meil.

$$
\text { Sant-Vincens. }{ }^{8} \text { ) }
$$

Ist ein dorff von vier heusern, hat doch gutte und wollversorgtte beherberunge.

1) Captieux. 2) Mont-de-Marsan.

9) Johann von Navarra, im Jahre 1512 durch Ferdinand von Aragonien vertrieben.

$\begin{array}{llll}\text { ग) 21. Mai. B) Dax. } & \text { 7dour. } & \text { 7) Mai. } & \text { 8) St. Vincent de Tyrosse. }\end{array}$ 


\section{Das Land Peschaya. ${ }^{1}$ ) \\ Bagonia.}

Ist ein haubtstat des Landts Pischaye, nohent an dem merhe gelegen, in der grosse Amberg gleichmessig, durch welche fleust ein groß wasser mit dem merch vermischet, in welchem man treffliche gutte Lechs und karpffen und ander vischs fänget. Aldo haben wir ein karpffen von XXXV pfunden faist und schmackhafftig umb zwaintzig Crutzer und ein Salma von XL pfunden umb 1 fl. gekaufft.

Diser Stat Ambtman ist unserm gnedigen herren entgegen geritten und die herrn des Raths in rotten kappen, wie die doctores tragen, haben auch, an der prucke vor der Stat versamlet, meinen gnedigen herren erlich entpfangen und nachvolgendt von allen thurmben schlangen und haubtstukke abgeschossen.

\section{Pyrenei-montes.}

3 meil.

Anyou. $\left.{ }^{2}\right)$

XXVI. May.

Ist ein klains dorff, in wellichem sich endet Franckreich und das gebiet des koniges von Franckreich, und was hernach volget, ist Hyspanien zugehorig und zustendig.

\section{Das Landt Bascho.}
4 meil.
Elysand 0. ${ }^{8}$ )
XXVII. may.

Ist auch ein dorff an dem pampalonischen gepirge gelegen, welches man Lateinischs Pyreneos montes nennet; do muß man etliche berge ain halbe meyle hoch, auch einer meylen hoch zwen tage überreitten, weliche on etlichen ortten gar unmöglich zureutten seint. ${ }^{4}$ )

In disem obgemeltten gepirge leydt das Landt Baschko, wellichs ein unhofflich volck hat, eine sunderliche sproche, welche mit dem welischen Latein, frantzosischen, deutschen und hispanischen nichts gemaynes hat, darin die Junckfrauen alle

1) Biscaya. 2) Ainhoue. 3) Elizondo.

1) Wie Leodius (S. 101 a) nitteilt, wähite Pfalzgraf Friedrich diesen Weg durch das unwirtliche Gebirge, weil er vermeiden wollte, seiner einstigen Jugendgeliebten, der verwitweten Königin Eleonore von Portugal, der Braut Franz' I. von Frankreich, der ältesten Schwester Kaiser Karls V., zu begegnen. 
beschoren seyndt kolbith ${ }^{1}$ ) und nach der paucken singen zum tantze, und an dem tantze zuspringen und alle geradigkheit $\mathrm{zu}$ uben, auch des pales $z u$ spielen ist den pristern unverweißlich. ${ }^{2}$ ) Diese obgemeltte Junckfrauen mit den henden an enander geschlossen und nach der paucken singende in den dorffern verhalden den Reuttern die Strosse und begeren von in eine verehrunge. Auch hat diß Landt sonderlich ungelerte priester, ${ }^{8}$ ) welchen die weyber, so sie auß der kirchen geen, die hende kussen, und in der kirchen offte den sauen an der Cassell. ${ }^{4}$ )
4 meil.
Alantza. $\left.{ }^{5}\right)$
XXVIII. May.

Ist in dem obgemelten gepirge auch ein dorff, in welchem der pfarrher am Sontage trinitatis ${ }^{6}$ ) zu dem umbgange sang Salva Regina und in der kirche zu einer ziere nichts dan Tischstucher und hanntzwehel ${ }^{7}$ ) hatt auffgehangen.

\section{Konigreich Navarr.}

3 meil.

Pampalona.

Ist ein $z y[m] l i c h e$ raynkliche stat, grosser dan Amberg, welliche des konigs von Navar gewest ist, darinne noch des kaysers kriegsleute ligen, welchen er uber XXII monadts soldts schuldig ist; haben uns zum fruestucke geladen und alle Erhe erzaiget.

Dieses obgemelts Landt hatt dises kaysers vatter konig Philips dem konige von Navar $^{8}$ ) genomen, ${ }^{9}$ ) welcher sich noch an des konigs von Franckreichs hoff stettiglich erhäldet.

1) kolbith (gewōhnlich "kolbicht”, "kolbig“; bayrisch : "kolbeth")= glatt geschoren. Vgl. Grimm: Deutsches Wörterbuch. V, 1611; v. kolbicht: 4a, sowie ebenda $1607 \mathrm{v}$. kolbe: 9.

2) Die Beteiligung an öffentlichen Spiclen war den Geistlichen bekanntlich verboten.

9) Vgl. L. v. Rozmital a. a. O. S. 166: "In dem land haben die pfaffen weiber und sein übel gelert und predigen auch uichts dan die zehen gebot und iederman bciclitet kein andre beicht, dann die der priester vorm altar spricht. Er hab groß oder klein sünd gethuen, so nent er doch keine mit namen, sunder mit der beicht wil er's ausgerichtet haben."

4) Saum an der Casel; casula, vestis sacerdotalis; Grimm a. a. O. III, 608.

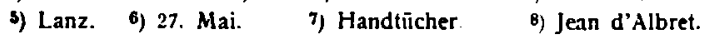
im Jahre 1512 Navarra. 
Die tagebuchartigen Aufzeichnungen des Dr. Johannes Lange. 413

4 meil.

Varasonia. ${ }^{1}$ )

XXIX. May.

Ist ein zimlich dorff auff einer höhe gelegen, auff welches kirchoff vast vil Cucumer asinium ${ }^{2}$ ) wechst.

4 meil.

Taffallia.

XXX. May.

Ist ein klayne stat, hat ein sloB, grosser und weytter dan die gantze stat, welcher haubtmann meinem gnedigen herren ist entgegen gerithen und eingeladen und vill erhe ertzaiget.

In diser Stat hat des doctor Lembergers pferdt nach essens auB einem sienden wasser, darzu slangen und frösche lyeffen, sich $\mathrm{zu}$ rehe $^{3}$ ). getruncken und $4 \mathrm{myl}$ darauff gegangen.

4 meyl.

Peraltha.

Ist ein klains stetlein an einem großen wasser ${ }^{4}$ ) und stainigem berge gelegen.

7 meil.

\section{Serviera. $\left.{ }^{3}\right)$}

Dißes Stetlein ist eyne lange gosse, an einem hohen berge gelegen, hat im tall ein kleyns flyessende wasser ${ }^{\sigma}$ ) und.vil feygenbäume. Aldo sein wir am tage Corporis Christi 7) still gelegen, do haben die Burger in weyBen hembden mit gemaltten rayffen vor dem Sacrament nach dem altten judischen gebrauch getantzet und gesprungen.

Auf disem wege drey meylen nach Paraltha fleusset das berumbt wasser Yberus, durch welches wir mit den pferden und Eseln gerytten sein, und ich, doctor Lemberger, mit meinem gnedigen herrn ubergefaren. An disem wasser endet sich das konigkreich Navar und fanget an Castilia.

\section{Castillia Konigkreich.}

4 meil.

Matelebreres. ${ }^{8}$ )

In diesem dorff haben sie den halben tag circuirt oder circuitum gehalden, und Gott mit schreyender stymbe umb regen, wasser und barmhertzigkeit gebetten, und altte Menner, auch

1) Barasoain. ク) Die Spring-, Spritz- oder Eselsgurke (Ecballium elaterium). $\begin{array}{llll}\text { 3) rehe, rāhe }=\text { Steifheit, nur von Tieren, besonders von Pferden. } & \text { 9) Arga. } & \text { 3) Cervera } \\ \text { del Rio Alhama. } & \text { 9) Rio Alhama. } & \text { 7) } 31 . \text { Mai. } & \text { 8) Matalebreras. }\end{array}$ 
knaben, Junckfrauen und kynder nacket und parfue $\beta$ gegangen und sich mit gayseln gehauen.

5 meil.

Gomora.

Ist ein kleines Stetlein, darin wir seint von einem pfaffen beherberget, welcher von meinem gnedigen herren versicherunge forderthe, das im nichts empfrembt wurde auB seinem Casten, die er in meines G. H. kamer hāthe. Alhie hat man kain brot zu verkauffen gefunden, sunder vor uns auB bevelch der herrschafft sonderlich pagen, wie dan auch zu Serviera und in andern nachvolgende Stetlein offt gescheen ist.

In diser Lantschafft hats in funff Monadtten nit geregnet, derhalben umb wasser grosser mangel waß allenthalben.

4 meil.

Maron. ${ }^{1}$

III. Juny.

Ist ein dorff unter einem Slosse gelegen, in wellichem ein weib das ander auf der gassen gefangen nam von wegen des weins, den sie uns gestolen hetten und die Justicia anrueffen, und der ungetreue wirth von uns forderet ainen silbern becher, den wir in seiner kamer widerfunden.")

2 meil. Fontha willa.

Ist ein klains dorff, auf deutschs zu dem morgenbrunlein genant, ${ }^{8}$ ) und habe doch mangel an wasser gehabt, der bronnen stet hinder dem dorff im grunde.

6 meil.

Reoffrio.

Ist ein zimlich dorff in einem grunde gelegen, aldo hat nach zukunfft meines gnedigen herrn got einen grossen Regen dem Armen volck verlihen, darumb sagetten, got het unsern herrn zu yn geschickt mit einem fruchtbaren regen. Diß dorff ist des Marckgraven von Nassa. $\left.{ }^{4}\right)$

\footnotetext{
1) Morún.

2) Leodius erzählt (S. 103f.) dieses an sich recht harmlose Ereignis sehr umständlich.

9) Wie der Vetfasser zu dieser Deutung kommt, vermag ich nicht anzugeben: fuente die Quelle; villa das Landgut, die kleine Stadt. Leodius (S. 108) nennt den Ort "Toutafilla pagus", was uns aber auch der Deutung nicht nāhler bringt.

9) Graf Heinrich von Nassau. Er war seit Juni 1524 in dritter Elie mit Menzia Zenette aus den Hause der Mendoza vermāhlt und dadurch Besitzer großer Liegenschaften in Spanien. Vgl. Meinardus: Der Katzenellenbogensche Erbfolgestreit $I_{1}, 78 \mathrm{f}$; eine Beschreibung der glänzenden Hochzeitsfeierlichkeiten ebenda $I_{21} 121 \mathrm{ff}$.
} 
Item von Pampilana baß gegen Schedrack seintht wir tegelich zwuschen Roßmaryn, Lavendel und Salve-Segelbaum geriten, und wo dise obgemeltte kreutter wachsen, do habe wir dorre und unfruchtbars stayniges Landt funden. Derhalben soll Teutsch nacion dise wolschmeckende kreutter vor ir graß und thanzepffen kains wechssels begeren.

4 meil.

Schedrack. ${ }^{1}$ )

V. Juny.

Ist ein groB dorff des Marggraven von Nassa, in welchem wir ain ducaten für einen wasserhaffen muessen $\mathrm{zu}$ pfandt geben, und sein von einem pfaffen beherberget worden, der alles vor uns geflohet hete und den gartten verschlossen, in wellichem wir die ersten reyffen opffel funden haben.

3 meil.

Hyta. $\left.{ }^{2}\right)$

Ist ein klains Stetlein, darin wir yn eines briesters haus sein beherbergert worden; hat auch ein schloß auff dem berge.

\section{4 meil. \\ Ouadalashara. ${ }^{8}$ ) \\ VI. Juny.}

Ist ein Stat grosser dan Amberg, dem hertzogen von guadalaschara ${ }^{4}$ ) zugehorig, hat vill Ölbaume und zimliche weinwachs.

3 meil.

Sant Türckas.

Ist ein ziemlich groB dorff, welches mit sambt einem ander ain doctor der Ertznei besoldet und vil grosser weynhäffen machet, einen umb ain ducaten, auch anderthalben.

In disem obgemeltten Stetlein und Stetten Castilie und Navarie seint weichsel feygen und allerlay getrayde, Gerste und korn, umb Corporis $\mathrm{Christi}^{5}$ ) reyff gewest und abgeschnitten, welches sie nicht außdreschen, sonder mit Eseln, Ochsen und pferden, die ein predt vol spitziger eingeschlagen steine daruber furen und schleppen, also außtretten, das das stroe allayne glidslang pleibet, derhalben pferde und Rinder kain stroe haben.

1) ladraque. $\quad$ 2) Wahrscheinlich Humánes. $\quad$ 9) Guadalajara.

1) Die Herzogswürde von Guadalajara war erblich in der Familie der Mendoza.

5) 31. Mai. 
4 meil.

Valdelagunna.

VII. Juny.

Ist ein klains dorff, in welchem der Edelman meinem gnedigen herrn beherbergete und von einer swartzen Morin vil kinder ine zuverkauffen auffzeuet; hat sunderlichen großen weinwachs.

In gantz Hisponia die Reichen und die Edlleut die swartze verkaufftte moryn und leybaygen haben, vergonnen yderman die fleyschlich zu erkennen, also doch das die frucht des herren beleyben syndt, welche er $\mathrm{ym}$ sibenden und zehenden Jare, auch Eltter umb XVI oder zwaintzig ducaten, auch vill teurer verkauffet.

5 meil.

Octavia. $\left.{ }^{1}\right)$

Ist ein Stat in der grosse fast Nurmberg gleichmessig des Bischoffs von Tholeth, in welcher ein edelman meinem gnedigen herren erbarlichen beherberget hatte, und der Ambtman meinen gnedigen herren auff den Abendt zu Gaste gehabt des andern tages, in welchem wir synt still gelegen, und mein gnediger herr, der von Haydeck, ich und zwen knaben haben yn einem weynhaffen ein volbadt gehabt und wol den sweiß abgewaschen.

Item Navar und Castilia und fast gantz Hisponia hat an holtz so grossen mangel, das sie iren wein in erden heffen behalten mussen, welcher einer $1 \%$ fuder weins heltet, auff die forme gemacht. ${ }^{2}$ ) Auch habe wir alle speyse mussen mit kleynen reyssen syden und brothen und das offte das schwerlichen bekomen mogen.

Item drey meilen nach Valdelagunna seindt wir uber das berumpte wasser Lateinisch Tagus genant geschifft, in welchem vortzeitten man vil Goldes gefunden hat. ${ }^{3}$ )

1) Ocaña.

2) Am Rande eine ganz flüchtige Zeichnung beigefügt.

3) Vgl. Lübker: Reallexikon des klassischen Altertums, Leipzig 1891, S. 1177: "(Der Tagus) führte nach den Berichten der Alten viel Goldsand mit sich, wovon sich jetzt nur geringe Spuren zeigen.“ Vgl. hierzu Dillon: Reise durch Spanien, Leipzig 1782, I, $256 \mathrm{f}$. Z Zahlreiche Literaturangaben über Ooldvorkommen im Tagus bei den alten Schriftstellerm findet man verzeichnet bei Pauly: Realenzyklopādie des klassischen Altertums, Stuttgart 1852, Bd. VI, Sp. 1581. 
5 meil.

$$
\text { Templeck. }{ }^{1} \text { ) }
$$

$X$. Juny.

Ist ein offen marck, in welchem unser wirth von wegen der Marranischen ${ }^{2}$ ) Secth verbrant waB und sein nomen auff ein gelbes tuch grob geschriben in die kirche gehenckt.

Merck! so yndert ein person, weyb oder man, wirtt bey dem Richtter bey dem Eyde beschuldiget, auch in irem abwesen, das sie der Marannischen ader Judischen secth anhennig sein, so wirt die beschuldigt person in abwesen des heymlichen zeugen gefordert, und so sie des gesteeht, so hatt die beschuldigtte person hab und all ir gutt verwircket, das dem richtter und der obrikheit haimfallet. So aber obgemeltte beclagtte person das laucknet, so hatt sy auch alles gut verlorn und auff gethonen Aydt des haymlichen anclagers wirt sie verbrent und ir Namen auff ein gelbs tuch, ayner elenn prayt und lanck, grob geschriben in die kirche an einer schnur uffgehangen, darumb sicht man vast in allen kirchen Hisponie zwaintzig, auch 40 und 70 tucher hangen.

Durch dise Jurisdiction werden vil rechtter Leute umb neudes und guts willen beclagt und auff falschen Aydt des anclagers und geytz des Richters leybs und guts beraubet, derhalben Nyemandts in Hispania wider die Geystlichen reden oder der Lutteryschen und Evangelischen sache one ferlikhait seines lebens gedencken [darf].

5 meil.

Villafranck.

XI. Juny.

Ist ein zymlich dorff, do sein wir zu mittage am XI. Juny gelegen und gezogen gegen Willaharta.

3 meil.

Willaharta.

Ist ein zimlich dorff, in welchem sich unser wirth vor funff tagen hăt lassen tauffen. In diser tagraysse seynd von hitze und starckes weins halben uff dem wege die nacht blyben ligen Hans Eseltreyber mit seinem gesellen und Arnolt, Koch, am grymen, ${ }^{8}$ ) und Vintzents und Gregorius auch kranck worden; derhalben sein wir ein tag still gelegen.

1) Tembleque.

2) Maranen, ein Schimpfwort der Spanier fūr getaufte, aber ihrer Religion im geheimen treu gebliebene Juden und Mauren.

3) Leibweh, Darmkolik. - Colica, daz krimmen (grimmen). 
5 meil.

Mantzanares.

Ist ein dorff, hatt auch weinwachs. In Castilia in vil dorffern und stetten ist verpotten die milch zu verkauffen, auff das sie die junge rynder und kelber also vil lenger lassen saugen und starck grosse ochssen auffziehen, mit welchen sie das veldt pauen und faren; haben schene horner, aynes Elbogen lang und drithalber Spanne von einander gewachssen mit den spitzen, und halden das vieche gantz sauber.

3 meil. Valdepenies. $\left.{ }^{1}\right)$

Ist ein zimlich dorff, welches seer ein grosse weinwachs hat; aldo ist mein gnediger herr vom Amptman Sannt Jacobs ${ }^{*}$ ) orden erhafftig beherbergt.

Alhie anfencklich muß man wein, brott, fleisch und auch fuetter mit sich furen, dan auff disem nachvolgende wegk etliche meylen findet man weder dorffer, noch stet, sunder allaine etliche heuser auffs veldt von wegen der wanderleute und kauffleute gebauet, darin man auch kain bethe oder koche heldet. Dise herbergen hispanischs nennet man ventas.

5 meilen.

Venta le rueleos.

Ist ein eintzig dorff zwuschen grossen bergen gelegen, aldo seindt wir auff der erden und etzliche auff der kaufleutte wolsecken gelegen.

Finis Castilie.

\section{Cattalonia konigreich.}

6 meil. Vilschis.

Ist ein kleins Stetlein uff einem hochen berge gelegen. ${ }^{8}$ ) In disem flecken muß man 5 meylen uber grosses gepirge reyten gantz staynigen und eben ${ }^{4}$ ) weck, der do nit all enden zu reytten ist.

Item [in] gantz Hisponien ist yderman freye, das wiltproth allerley $z u$ schiessen, welches die pauern sunderlich in disen

1) Valdepeñas.

2) Orden des heiligen Jakob vom Schwert, spanischer Militärorden.

s) Vilches: "malerisch zwischen zwei Bergen gelegen" (Baedeker: Spanien und Portugal S. 312).

) Soll wohl statt und eben uneben heißen.

D. Red. 
pergen mit vergyfftten klaynen pfeylen schyessen, welche gifft also starck ist, das sie den menschen todet, so allain des menschen pluet oder klaynes wundlein darmit bestrichen wirt. ${ }^{1}$ )

4 meil.

Ubyda. $\left.{ }^{2}\right)$

Ist ein Stat Amberg in der grosse gleichmessig, hat vil wein und getraidt und Mauelberbaum und auff dem velde drey meylen lang und sunderlich an dem wege wachsen seer vill Capren. Auff disem wege zwue meylen noch Vilschis im grunde am wasser hat der Jobst Brantner, Ragas Steffan, Wastel Barbirer und Bock ain ainem Steige verstossen, den hat auB bevelch meines gnedigen herren der Bock nachgerent und sein pferdt verderbet und ist auch schwerlich widerfunden. ${ }^{8}$ )

3 meil.

Schoda. $\left.{ }^{4}\right)$

Ist ein dorfflein, in welchem wir die pferde nicht woll haben konnen gestellen, und ain meyll hinder dem dorfflein fenget sich ain gepirischs landt an paB gegen Granaten, und uberall auff den bergen sicht man klayne thumlein und warthen von den morischen gebauethet, ${ }^{6}$ ) darvon sie an einander beruffen und Irer vheinde zukunfft verkondiget haben.

4 meil. Venta Karafaschall.

Ist ain aintzick hauB, darinna(!) wir nicht alle haben kunnen stellen und ayn tayls auff der erden im hause, die andern ym velde gelegen. Aldo ist der Marschalck mit seinem knechte und Gregorio ${ }^{6}$ ) und dem Lehendell (?) gegen Granathen von Vuida ? geritten und hat durch des kaysers bevelche uns herberge

1) Über diese vergifteten Pfeile, welche die Mauren auch im Kampf gegen die Christen verwandten, vgl. Prescott: Geschichte Ferdinands und Isabellas I, 390.

\%) Ubeda.

3) Ein Reiseabenteuer, dessen tatsächlicher Kern in dieser knappen Schilderung nicht recht deutlich zu erkennen ist.

4) lódar.

5) Vgl. Prescott: Ferdinand und Isabella (deutsche Ausgabe) I, 387: „... innerhalb der Grenzen Granadas gab es ... zehnmal mehr feste Plätze, als jetzt in der ganzen Halbinsel. Sie standen auf dem Kamm irgend eines Abgrundes oder einer steilen sierra, deren natūrliche Stärke noch durch das feste Mauerwerk vermehrt wurde, von dem sie umgeben waren."

b) Gregorius Mayer, Silberschließer.

7) Vgl. Leodius S. 107a: [Marescallus], quem ex Ubeda praemiserat Princeps Granatam, ut nobis de hospitiis prospiceret." 
bestellen muessen, und an des kaysers vorbitte und bevelch het er uns kain herberge konnen bestellen.

6 meil. Guadalia-Horruna.

Ist ein dorff durch konig Ferdinandum von wegen der Rauber, die sich in dem obgemeltten gepirge erhalden haben, gebauet. Aldo macht man schone und wolgeferbte gleser.

4 meil.

Asanalios.

Ist ein dorff, darinne wir haben uff die herberge zu Granathen zu bestellen 3 tage gewartet. Ist der Morischken gewest, darinne man noch ir schlos zurbrochen sicht uff einem berge.

4 meil.

Allabalath.

Ist ain dorff ein halbe meil von Granathen gelegen, in wellichem, so wir auB kayserlichem bevelch herberge eingenomen hatte, ist der wirth von Granathen selb dritthe komen mit speyße und uns außzutreiben im furgenomen.

\section{Das Konigreich Granaten.}

\section{Granaten. $\left.{ }^{1}\right)$}

Ist ein Stat eines namens zwuschen den bergen also gelegen, das man die von kaynem eusserlichen berge aber orte gantz besehen kann. Ist vast zway Nurmberg groB, und auff den eusserlichen bergen vindet man in den allerhaisten tagen vill schnees, ${ }^{2}$ ) darmit man den wein kulet. Dise stat leyt nicht zwolff meylen von dem mittelmer, also das man darau $\beta$ in 3 tagen mag in Affrica sein, und vier Tagen am ende der welt und nydergangs.

Dise stat ist der weyssen moren gewest und hott zwen konige $^{3}$ ) gehabt, vor welcher konig Verdinandus hat sechs Jar ${ }^{4}$ ) gelegen und ein Stetlein mit seinem here Santha Fede genant ${ }^{b}$ )

1) Am 23. Juni kam Pfalzgraf Friedrich in Granada an. Vgl. Alex. Schweiss an Graf Wilhelm von Nassau, Granada, 25. Juni 1526: „Pfaltzgraf Fridreich ist fur zweien tagen hie bey $k . m$. ankoinen“ (Meinardus: Der Katzenellenbogensche Erbfolgestreit $I_{2}, 182$ ). Seit dem 4. Juni weilte der Kaiser in Granada. (Forschungen zur deutschen Geschichte Bd. V.)

2) In der südöstlich von Granada gelegenen Sierra Nevada.

3) Boabdil und nach Abul Hassans Tode (1485) Es Sagall, mder Recke."

-) Sechs Jahre währte der ganze Krieg um Granada, nicht aber die Belagerung der Stadt. b) Binnen acht Wochen im Spätherbst des Jahres 1491. - Wenige Monate spāier ergab sich Granada. 
gebauet, ${ }^{1}$ ) und nochvolgens im 7 . Jare von der kunigin Eltzabeth gewunnen und Christen worden.

Item das halb tayll diser Stat volcks sein weysse moren, welcher weyber und junckfrawen alle weysse schyffhosen und ploderthe ${ }^{2}$ ) antragen, und das haubt und leib mit einem weyssen tuche, vast wie bey uns die dorffhirtten, beclaydet $\mathrm{paB}$ auff die waden, und das Tuch vorne alle fur das halbe Antlitz halden, und das dise klayde mogen ine nachgelassen und freyer seie, muB ein Jetzliche person dem kayser darvon jerlichen ain ducaten geben, und welche am Sontage die predige versaumet, dem pfarher ein Reall. In diser Stat an den bergen sicht man noch tiffe gruben, in welchen die gefangen Cristen mit sambt einem Bischoff des nachts geschlossen und am tage $z u$ allerlay Arbait vermyet und gebraucht sein worden.

Item dise obgemeltte Stat ist an sancth Johans tage ? erobert, 9 derhalben sie jerlichen an dem selbigen tage des morgen frue die Edelleut und Burger auff Morischkhen und Turckgsche art mit schilden und lantzen gerust vor der Stat ein scharmitzel halden und einen triumph nach essens; so lassen sye auff dem marckthe sechs oder siben ost[!] ochsen dem gemaynen man jagen und stechen, darnach komen die Raysigen auff turckischs und Morischkisch zu rosse gerust und in zway tayll getayllet, schiessen mit schweren dicken ruern uff einander und ein tayl umbs andere begibt sich die fluecht und stellet sich wider $z u$ der were.

Dises Spiel habe wir den kayser zu Granaten in aigner person und gegenwurt der kayserin mit irem porthugalischen

1) Vgl. hierzu Prescott: Ferdinand und Isabella, Leipzig 1842, I, 481 : „Die Stadt hatte eine viereckige Gestalt und war mit zwei geräumigen Zngangen versehen, die sich in der Mitte rechtwinkelig durchschnitten, in der Form eines Kreuzes, an dessen vier äußersten Enden sich stattliche Tore befanden. ... Als sie fertig war, wünschte das ganze Heer, die neue Stadt mōchte den Namen seiner berühmten Kōnigin erhalten; doch Isabella lehate diese Huldigung bescheiden ab und gab dem Orte den Namen Santa - Fé."

2) Abgeleitet von nblodern “ [= fluere, laxum esse, bauschen]; vgl. Orimm : Deutsches Wörterbuch II, 141.

3) 3. Januar 1492.

4) Diese Notiz ist nicht ganz genau: die Bedingungen zur Übergabe wurden von Ferdinand und Isabella bestātigh am 25 . November 1491, die Übergabe selbst und der feierliche Einzug erfolgte am 2. Januar 1492; vgl. Prescott a. a. O. S. 482 f. sowie S. 486, auch Anm. 20. 
frauenzimer zu Granathen an sannt Johanns tage ') halden [sehen], in welchem drey menner von den Ochsen seint auff den tode verwundt worden und ein gaul mit einem Ror auff das haubt geschossen, ist also balde nydergefallen und auff der stat belieben.

Item den obgemeltten Moren seint allerlay werhee bey in zu tragen, sie wandern dan uber felt, oder in irem hause $\mathrm{zu}$ halden bey grosser pene verboten, auBgeschlossen ein klaines protmesser und ein fleyschmesser, darmit sie zuhauen, welches an ein kethen gefast ist, derhalben die Obrikheit alle viertzehen tage ihre heuser lasset besuchen.

Item auff den letzten tage zu Granathen hat der kayser meinen Gnedigen herren in den gartten unter dem schlosse ${ }^{2}$ ) gelegen zu besichtigen den Morischken tantz gefuret, welche alle mit sunderlichen gutten Perlein und edelm gestaine umb die Oren, Stirne und Arme getziret und geclaydet, fast wie bey der messe dyaconi, auff ires Landes art getantzt haben nach der Lautten, geygen und paucken, auff welchen 3 weyber bey funffzig, auch eine umb die viertzig jare alt gespilet haben und mit heBlicher pauerischen stymme darunder gesungen und etliche die hende ineinander $z u$ frolocken geschlagen.

Am ende des tantz seindt auff einen berg komen Morischken weyber und haben sich mit außstrackten baynen uff einem sayle an zwyn nußbaueme geknopfft gegen dem kayser geschackelt und gerötzschet und auff ir sproche geschrien: wer wol lebet alhie, der feret allso in den Himel. Noch disem tantze hatt man yn wasser $\mathrm{zu}$ trincken gegeben.

Item die weyssen moren und junckfrauen in Castilia ferben [mit] gelbfarbe die Negeln an den fingern, wie bey uns die Gerber, welches sie halden für ein sunderliche zier, und ist einer Junckfrauen ein grosse schande, wann sie wein truncke, der halben sie alle wasser trincken.

In der obgemelten Stat Granathen macht man allerlay seyden gewandt, sonder ausserhalben schwartz, keines von ander bestendiger farbe und weniger oder nichts wolfayler dan in deutschen

1) 24. Juni : Joannis baptiste. - Der Verfasser wie auch Leodius, S. 110 a, nehmen an, Granada sei am 24. Juni gefallen.

2) Die Alhambra. 
Landen, außgenomen döpel Taffath, der so [do? D. Red.] seer wolgemacht ist; und perlein seindt auch do wolfayl.

Umb die stat ist auch ein grosser lustiger weingarthe und weinbachs; die ersten zwen tage haben wir kain bethe in der Stat konnen bekomen und auff der erden gelegen, darnach haben wir bethgewandt von den weyssen moren bestelt, darumb wir in funffzehen ducaten haben müssen verpfenden. Sein $1+$ tag zu Granatten gelegen und am 7. tag July in dem namen gottes mit freuden widerumb gekeret.

Item des kaysers schloB ist von den Morischken auff einen bergk in der Stat gebauet, darinne man noch sicht die lustige und kunstenreiche bade ${ }^{1}$ ) des Morischken koniges, in welchem er mit seinen beybern gebadet hat, welcher er dann vil nach seinem wolgefallen gehabt hat; und welche er dan noch dem bade begert hat, der hat er ainen Apffel zugeschickt.

Aus disem obgemeltten schlosse, darinne auch ain weyer ist, ') fleysset das wasser vast durch alle namhafftige heuser der statt Granathen. ${ }^{\text {) }}$ Ist ein ungesunt wasser, darvon man die Rure lyderlich uberkomet, und haben kain ander wasser, auch kainen brunnen.

\section{Reditus ader widerzug von Granathen am Sibenden Tag July} angefangen, wie nachvolgeth.
1 meil.
Albaloth.
VI. July.

Am VI. tage des Monadts July ist mein Gnediger herr von Granathen zu dem graven von Nassau in sein schloB, Allakalahorra ') genant, geritten und wider zu Ubida zu uns komen;

1) In seinen Epistolae medicinales (Basel 1554) S. 184 stellt unser Verfasser diese Băder als vorbildlich hin. .... quis balneorum in Galliis et Romae fragmenta et vetus etiam illud in Hispaniis Granati regum Mauritaniae balneum in arce Alhambre viderit, ad illorum normam construere possit. 4

z) Der sog. "Myrtenhof“.

s) Vgl. dazu Leodius S. 111 a: „Sexta [sc. res admiranda Oranada's], est Darrus amnis seu torrens, qui septendecem millibus passuum ab urbe ex alto iugo montis ortus, omnibus fere civitatis domibus aquas abunde praebet, et saluberrimas esse dicunt, licet aliter deprehendimus."

4) Calahorra am Almeria, östlich von Granada (selbstverstāndlich nicht Calahorra am Ebro, wie Meiuardus: Der Katzenellenbogensche Erbfolgestreit $I_{1}, 79$ meint); vgl. die begeisterte Schilderung dieses Schlosses durch den nassauischen Sekretär Alexander SchweiB : "und was mir hertzlichen lieb, das der pfaltzgraf dohin kam, allein nur solch haus, wars sunst umb anders nichts willen gewesen war, auch zu besehen. Wand ich sag e. g. zu, 
sunder wir den 7. Tag July auff den Abent seindt paB gegen Albaloth geritten und nachvolgens die altte strasse biB zu der venta le ruelleos.

3 meil.

Venta Le Ruelleos.

Aldo in disem dorff habe wir kärrhen gemuet und wein und Speis mit uns gefuret von wegen der pōßen zukunfftigen herbergen.

4 meil.

Venta de Canales.

Ist ein aintzigs hauß in einem wuesten veldt gebauet, dohin seindt wir uber einen feur[!] staynigen weg gefaren und nicht also vil wassers bekomen, daB wir die pferde und Esel hetten konnen nach notdurfit trencken.

\section{5 meil. \\ Alamacra.}

Ist ein Stetlein dem Neuenmarck gleichmessig, darinne uns die Fucker beherberget und alle erhe ertzaigetten. Aldo ${ }^{1}$ ) haben die Fucker des kaysers und etlicher ortten Hisponie zehenet bestanden, darvon sie sich betzalen von des kaysers wegen.

Auff disem wege zwu meylen von der obgemeltte venta schepfft man an einem Rade mit einem Esel wasser, das verkaufft man den Eselln und pferden und menschen ain trunck umb ayn heller.

5 meil.

Melagon.

Ist ein dorff nit groß auf disem wege. Ist bey einer Meile ein zerbrochen sloB, bey welchem das vadianum, das siben meil under der erden fleust, ${ }^{2}$ ) wider herfur an Tag entspringet. Ist ein clar wasser und hat doch einen bösen Rauch.

das ich vil hübscher hern heuser in Hispanien gesehen, aber noch keins so lustig, auch reich von mermelsteynen, seulen, stiegen und anderm und sunderlich von so guten ordinantien, als das, das auch mit seinen vier thurmen umbher und guten vestungen und geschutz nach dieser landart wol versehn und an allem nichts gespart ist. ... Ich hett gem gehabt, das m. g. h. e. g. das haus hett abryssen lassen und zugeschirkt, so meint sein g., es wer nit wol muglich, das es wol verstanden mocht werden." (Al. Schweiß an Graf Wilhelm von Nassau. Calahorra, 9. Juli 1526: Meinardus a. a. O. I 1 , 183).

1) In Almagro war der Sitz der Oeneralverwaltung der Fuggerschen Pachtung aus den Einkūnften der drei spanischen geistlichen Ritterorden Santiago, Alcantara und Calatrava. Zur Sache vgl. K. Häbler: Die Geschichte der Fuggerschen Handlung in Spanien (Weimar 1887) S. 72 ff., über die Niederlassung in Almagro ebenda S. $79 \mathrm{ff}$.

ク) Ouidiana, in Altertum Anas. Vgl. Pauly-Wissowa: Realenzyklopādie des klassischen Altertums (Stuttgart 1894) Bd. I \&, Sp. 2064: "Anas . . nimmt, nachdem er anfangs in einem regelmāßigen Bette, zuweilen unter der Erde sich verlierend, westwārts geströmt, eine sūdliche Richtung.“ 
Item mein $G$. $H$. hat seyden zu Granathen umb zehen ducaten gekaufft und sein gulden Paternoster achtzig gulden werdt zu Alamagra verlorn; die hat der Fucker diener here gegen Malagon und das paternoster gegen Tholeth uns nachgefuret und uberantwort, darumb in mein g. H. mit einem seyden wambes vereret hat.

+ meil.

Venta Sutanda.

Ist ein hauB, eytzlich in feldt gebauet, und so wir kain kamer noch bethe darinne gefunden haben, seyndt wir die gantze nacht vier Meylen gegen Jeuenes getzogen und auff dem velde gessen.

4 meil.

Jeuenes. ${ }^{1}$ )

Ist ein dorff, darinne man doch allerley hantwercksleut fyndet. Auff disem wege sein wir in der nacht durch einen Aquaductum gerithen, uff welchem das trinckwasser in die Stat Tholeth gefuret ist worden und auch in eine andere Stat uff der rechtten handt gelegen.

Item ain meyle vor der obgemeltten venta ist ein berg, an welchem sechstausent Piscayn haben 15 Tausent Moren erschlagen, darumb man noch auff dem berge all enden in den klaynen staynhauffen vil Creutze stecken sicht.

4 meil.

Zoffrinum.

Ist ein haimlich groß wolgepauets dorff, darinne wir gutte herberge gehabt haben, hat ein grosse weinwachs und holtz ein notdurfft.

Item in dem konigreich Navare und Castilia, auch sonderlich in Cattilonia von wegen Abbruch des Regens und wassers haben sie in den gerthen Bronne, darauff sie mit einem wasserradt den gantzen garten begissen, darmit sye in den heyssen monadten die fruchtbaren baume und pflantzen erhalden.

3 meil.

Tholeth.

Ist ein Stat fast also Nurmberg groß ongeverlich, hat drey zimliche berge, und daran fleusset das wasser Tagus genant; aldo sein die Thumbherren meinem G. $H$. entgegen gerythen,

1) Yébenes. 
und in eines thumbherrn hause, mit graß und bauen getzirt, beherberget, und vor essens des gestiffts kleynet und heylthumb, ${ }^{1}$ ) hundert tausent ducaten werth, getzaiget, weiches konig Ferdinandus und konig Ludwig auß Franckreich in von Canstantinopel zugeschickt hatt, des brieff und Sydel sie uns auch zaigten.

Diß gestiffts Bischoff hat jerlich achtzig tausent ducaten Rendt ") und ein kirchen, der gleichen mit zierheit der Capeln und gebeude ich in gantz Hisponia, Franckreich und in deutschen Landen nicht gesehen habe; hat $\mathrm{zu}$ erhaldunge des gebeudes jerlichs Rendt 10 tausent ducaten.

Item in diser Stat ist mein gnediger h. mit sambt dem herren von Haydeck, Jobsten Prantner, Santi Marie und Bastel Barbirer die post auff den Reichstag gegen Speyer gerythen, und ich bin mit sambt dem Marschalck und andern gesynde diße nochgeschriben weckh getzogen. In diser Stat ist gantz bose trinckwasser und seer boser geschwilder lufft; darin man vor tzeitten offeliche freue schulen der swartzen kunst gehalden hat.

6 meil.

Lyestkes. ${ }^{3}$ )

Ist ein zimlichs dorff, in welchem wir unser speysse und lichte vor des wirths dybischen tochtern nicht verhalden konden.

Diesen tag ist vast grosse hitze gewest, und des nachses[!] des marschalcks zeltter ym stal gestorben.

6 meil.

Matril.

Ist ein zimliche grosse stat, darinne der Moren konig ist gefangen gehaiden und jetz diser konig von Franckreich auch gegen neun monedt gefangen gehalden. Dise Stadt leydt mitten in Hisponien.

1) Wahrscheinlich die Custodia; vgl. über dieselbe Th. von Bernhardi: ReiseErinnerungen aus Spanien (Berlin 1886) S. 422: "Namentlich besitzt diese Kirche die berühmteste und größte silberne Custodia, die es überhaupt gibt, die ihres Umfanges wegen auseinandergenommen und verpackt aufbexahrt und nur zum Frohnleichnamsfest zusammengefügt wird; sie wird dann, wie man sagt, durch 80000 Schrauben zusammengehalten. Die Anweisung, wie die einzelnen Teile aneinander zu fügen sind, füllt einen kleinen Oktavband.“ - Über ein anderes besonderes Kleinod dieses Stiftes, das hier auch gemeint sein kann, vgl. Rozmital a. a. O. S. 187: „In der stat sahen wir sant Johans Baptistae haubt und vil kostlichs heiltum, und sahen die kostlichsten bibel, die man meint, die in der Cristenheit sey:“ - Über die Schătze der Kathedrale von Toledo insgesamt vgl. $M$. Willkomm: Wanderungen durch .... Spanien (Leipzig 1852) II, $306 \mathrm{ff}$.

y) Über die Einkünfte des Erzbischofs von Toledo vgl. Prescott: Ferdinand und Isabella I, $34 \mathrm{f}$. sowie II, 586, Anm. 14.

9) Illescas. 
7 meil.

Santl-Augustin.

Ist ein dorff, darin wir bosen wein und stincken wasser haben mussen tryncken, dan dem Esel, den wir mit 4 krogen ein halbe meyle nach wasser geschicket hetten, den hatte ein ander lediger esell umbgestossen und die wasser kruge zerbrochen.

7 meil.

Butrago. ')

Ist ein kleines Stetlein des hertzogen von Guadalaschara an einem wasser und lustigen gepirgen mit sambt dem eim wolgebauten schlosse gelegen; aldo haben wir an santh Jacobs ${ }^{2}$ ) abent fleisch gessen.

7 meil.

Busigillas. ${ }^{8}$ )

Ist ein dorff auff einem fruchtbaren und getraidraichen erbothen gelegen, do haben wir pōse wasser gehabt und gantz ein bose herberge und mangel am brothe.

Dise tagrayse ist durch einen heymlichen lustigen tall, auB welches berge vil gutter brunne entspringen, und das korn und gerste noch umb sannt Jacobs Tag bluet, sunst in gantz $\mathrm{Hy}$ spania all enden abgeschnitten und außgetroschen.

7 meil.

Aranda de Duro.

Ist ein Stat also groB alls der Neuenmargkt, dardurch ein grosses wassers, Duro genant, fleysset und hat grossen und fruchtbaren weinwachs und auch getraide.

7 meil.

Lermes.

Ist ein kleins Stetlein uff einem hohen berge gelegen, do man die grōnen vische ane wissen des pflegers nit verkauffen darff; aldo hin ist des vice Regis von Neapolis hoffgesinde auch alldohin komen.

7 meil.

Burgos.

Ist ein Stat in der groB Amberg, an einem wasser gelegen, daran ein grosser handel mit wolle ist, und hat in der Stat ein Bisthumb und wolgebauete kirchen mit gezincthen thurmben vast woll getziret; in welcher Stat der Contestabuli hat eine
1) Biatrago.
9) 25 . Juli.
3) Boceguillos. 
solche lustige wolgetzirte (apel $\left.{ }^{1}\right)$ gebauet, der gleich in $\mathrm{Hi}$ sponia, Franckreich, Italia und Germania nit befunden wirt.

Vor der Stat ist sanct Augustinkirche, in welcher in der Capel des Creutzganges stet ein Crucifix, welches auff dem wasser ein kauffman gefunden hat, und Nicodemus nach der gestalt Jhesu Christi am Creutze hangende soll geschnitten haben; ${ }^{2}$ ) hengen darbey vill wexerbilde.

AuB diser kirchen vor zwayen Jaren an heyligen Charfreytag ${ }^{8}$ ) ist ein puebfertiger sunder wallen gegangen und ain kreutz auff seinem Rucken getragen und under seinen fuessen, wo er gangen ist, ist das graß alles verbrant und verdorret: Nu rathe, wer mag das gewest sein? ${ }^{4}$ )

Item ein halbe meyle vor der stat ist ein koniglich hospitall, welches hat sechs tausent ducaten jerlichs Rendts, darvon etzliche Reysige edelleut in dem hospital wonende erhalden werden, und von dem uberigen alle Jacobsbruder gespeysset und beherberget ain nacht und die krancken, piB das sie genesen. Dises Spitals Spitalmayster hat mit sambt seiner tochter bey 500 Pilgramsleut mit gift getodet und ire Barschafft behalden; darumb er auch gericht worden ist mit sambt der tochter.

Dise stat leut auff der rechtten strosse zu sanct Jacob, ${ }^{6}$ ) darvon noch zu sannt Jacob gerechent wirt hundert meyl.

Item in einem dorfe von Burgos ain meyl an der strasse treibt man des morgens frue die khue bey dem kirchoffen durch ein stōle und besprengt der briester das viche mit geweihttem wasser.

8 meil.

$$
\text { Breineske. ? }
$$

Ist ein kleines Stetlein des Contestabuli, darinne der jüngste son des koniges von Franckreich, hertzog zu Orliens, ${ }^{7}$ ) gefangen wardt und mit Spissen und krichsleutten bewardt war.

1) Die große gotische "Capilla del Condestable" in der Kathedrale hinter dem Hochaltar, seit 1482 für den Connetable Pedro Hernandez de Velasco, Grafen von Haro, erbaut.

ๆ) Vgl. hienüber sehr ausfūhrlich Rozmital a. a. O. S. $168 \mathrm{ff}$.

э) 25. Mărz 1524.

4) Ich vermag leider nicht anzugeben, um wen es sich hier handelt.

5) San lago di Compostella.

$\eta$ Der spātere Kōnig Heinrich II. von Frankreich; er wie sein ālterer Bruder waren auf Grund des Friedens von Madrid an Kaiser Karl als Geiseln für die pūnktliche Durchführung der Bestimmungen ausgeliefert worden. 
Die tagebuchartigen Aufzeichnungen des Dr. Johannes Lange. 429

7 meil. Miranda.

Ist ein kleines Stetlein am wasser Ibero gelegen, darinne der elste Son des konigs $^{1}$ ) von Franckreich Delphin ${ }^{2}$ ) gefencklich bewart warhe.

\section{Pystkaya das Landt.}

5 meil.

Victoria.

$\mathrm{DiB}$ ist ein zimliche grosse stat, hat in einer meyle umb sich mer dan 60 dorffer ligende. Ist ein anfanck des landes Pischaye, welches zwuschen den bergen leydt. In einem tall darinne nichts mer dann Opffel uberflussiglich vil wachssent, darvon sie iren tranck machen, und vil eysenhemer haben und fast gantz Castilien mit eysen versorgen und Brittannia, ${ }^{8}$ ) welchem sie bey fonte Rabinie ${ }^{1}$ ) eysen umb korn und getraide uber das merhe zuschicken. DiB Landt hat schöne weibsbilder und beschorne kolbige Junckfrauen und ein sonderliche sproche, welche sich mit keines andern Landes sproche vermischt und vergleichet.

Item zu Victorie seint uns betrieglicher weyse paßbrieffe eingeret worden von der Stat obristen, und die wirthin hat den Marschalck für einen Juden gehalden und gescholtten, derhalben sie auch in anderthalben stunde uns kain brot wollte verkauffen.

5 meil.

Alharta.

Ein dorff under sannt Adrian berge gelegen, darinne wir schwartze wein und dicker dan die tinckte und sauer haben mussen trincken.

\section{Santh Adrian bergk. ${ }^{3}$ )}

Ist vast einer deutschen meylen hoch gantz boses steyniges weges, welcher schwarlich auff zu reyten ist und ungleich herab zu reyten. So man von der höhe herab zeyhet, so muB man durch einen auBgehorn (ader durchholertten) vels, großer dan ein zimlicher grosser keller, reyten, darinne man ein kleines Capellelein findet und auch wein sambt einer schonen wirthin.

1) Karl; gest. 1536. 2) Am Rande mit roter Tinte: „Der Deiphin“.

9) Bretagne. $\quad$ ) Fuenterrabia. 3) Sierra de San Adrian. 
3 meil.

Secura.

Ist ein kleins Stetlein under sannth Adrian berge gelegen. DiBe 8 meylen seint wir einen tag geritthen mit sonderlicher muehe; ich halts dafur, das in anderthalber meyle umb dise sta: mer Öpffel dan in meines gnedigen herren hertzogthumb wachssen.

5 meylen.

Tholosetha.

Auff disem wege hats vil Opffelbaum durch den gantzen tall und ein sehen mer, ${ }^{1}$ ) derhalben [man] auch in disem stetlein die besten hyspanischen spaden macht.

Auff disem wege sicht man offt in einem klaynen Acker acht hundert junger Öpffelbaume zwayer ellen hoch gepflantzet, und vast in vill gertten junge eschenbaume vom stamen biß in den gypffel beschnytten und ein dreytzehn ellen hoch und nicht dicker dan 5 aber vier, auch 7 vinger, darauß sie gantz veste und werhafftige Lantzen und knechstzspiesse machen in der Pyschkay und sich allayne der erbait etlicher hantwercksleute erneren.

Sant Marie de Rome und Fonteraui. ${ }^{2}$ )

Ist ein klaines dorff am eusern merhe gelegen, ein klaine halbe meyle von der Stat Fonterraui, welche am mereh leydt, nit vil grosser dan Sultzpach, welche der konig von Franckreich hat vor funff Jaren dem kayser abgewunnen, ${ }^{8}$ ) durch die deutzschen Landsknechtte, und uber zway jar hots der kayser mit den obgemeltten knechtten widergewonnen. ${ }^{4}$ )

In diser stat $\mathrm{zu}$ besichtigen, wie sie zuschossen und gewonnen sey, bin ich mit dem marschalck gefaren auff dem merehe, und weyl wir wider heimfuhren, ist das merhe nach seiner eigenschafft uns entwichen und das schiff uffgestanden, daz man uns ein teyl wegs hatt mussen auß dem wasser tragen, und des andertayls haben wir zu fuesse durch den schleym und kott muessen wathen. Uber dem wasser bei Sannt Marie fanget an Franckreich.

1) Die von mir gegebene Lesart ist zweifellos; den Sinn verstehe ich nicht. (Eisenhämmer. D. Red. Vgl. S. 429.)

2) Fuenterrabia. $\quad$ s) Im September 1521 durch den Admiral Bonnivet.

†) Im Februar 1524. 


\section{Principium regni Francie.}

An disem wasser leydt des kaysers schloB, darauff er etzliche knechtte heldt, die one paBbrieve des kajsers nyemandts in Franckreich lassen, und allein mussen die kaufleute alle ir gutter vertzollen.

Von den hispanischen herbergen und wirtthen.

Der diße obgemeltte strasse mit etzlichen pferden ader fueßknechtten in Hyspanien ziehen will, dem ist von notten, das er auß und ein zu reytten sicher gelayte habe des Konigs von franckreich, sunst wirt er an frontirn oder Grentzen gefencklich auffgehaltten, und auch von dem Kayser schryff[t]lichen und ernstlichen befel habe an alle stette und dorffer Hyspanie, das man im herberge schaffe und, weB er notdurfftig sey, umb ein zimlich geldt verkeuffe und mittheyle.

Zum andern das er an der frontzosischen grentz zu Bayona kauffe stuel, Tisch, häffen, brotspeis, kessel, Kellen und pfannen, waz man in der kuchen geprauchet, und uff einem Esel nachfure, dan in den Hisponischen Herbergen vindet mans nicht zu kauffen, noch zu entlehen, und so sie doch solchen obgemeltten haußroth hetthen, das do seltzam ist, so verleugnen sie den und verschliessen in. Auch findet man in obgemeltten herbergen kain stallunge, kain heue noch streue, auch weder roBbaren ${ }^{1}$ ) noch rayffe, sunder klein zerriben strohe glydtslang und gerste, auch waitze an stat des haberns, darmit man fuettert.

Das bethgewant ist nicht von federn, sunder mit wolle, etzlichs auch mit erbstroe auBgefüllet und die Leyloch sein von vast gutter und subtiler leynbath, welche sie mit waschen sauber und reyn halden, jedoch hātten on vil orten die leuse, wantzen und muckhen die herberge vor uns bestelt und eingenomen. Auch vindet man vast in allen heusern Hyspanie und sunderlich yn den herbergen kein haymlich gemach oder sprochheuslein, sunder yderman leufft in die stelle, darvon die stallunge also stincken, daz nicht wunder wer, das gestancks halbe die geulle verdurben. ${ }^{2}$ )

1) Krippen.

2) Vgl. hierzu Aløin Schultz: Das hãusliche Leben der europāischen Kulturvōlker vom Mittelalter bis zur zweiten Hälfte des 18. Jahrhunderts (München 1903) S. 25: "Bei 
So aber der haußwirth ein stathafftiger man ist, so holdt er zwe seue eingeschlossen, die lest er auff den abent den unflat uffessen, das er ym darmit auch einen nutz schaffete.

Item in den hisponischen herbergen vindel man seltten holtz zu kochen oder des brots ein notturfft, dan von wegen mangel des holtz helt man in den dorffern und stetlein einen gemaynen Ofen, darinne ein ytzlicher haußwirth ym nicht mer, dan er auff ein tag nottūrfftig ist, pachen lest, derhalben die obrikheit hat mussen offte schaffen, das man vor uns auch bueche. Ursache solcher bosen und ungebauten herbergen ist des Landes unfruchtbarkheit und das ein itzlicher burger in Castilia schuldig ist, die edelleute halb umbsunst zu beherbergen und in das halbe tayl ires hauB und hauBraths einzugeben, der halben die heuser nicht stathafftig gebauet werden. ${ }^{1}$ )

Item Hysponia ist ein seer birgiß Lande von gantz unfruchtbarn gebirge, hatt starcke ungebreuchliche weyne, weiche man in erdthefen haldet und in gezierhtten GeyBheutten überlandt furet, darnach sie alle schmecken, und dysse wein, so man sye trincket, mūessen das halbe ader drytthayl mit wasser gemischen[!], und von dem ersten Trunckhe, den einer trinckhet, bricht im von stund an der schweis uber den ganzen leyb auß.

Auch seint vast alle stett, heuser, thurme und Statmauern in Hysponia nicht von holtz oder Steine, sunder von gedurthen erdtkloßen, wie die ungebranthen ziegel gemacht, gebauet und geweyßset. Auch bedarff Hysponia nit sunderlich vest gebeue, dan mans des wassers und herberge und allerlay provandt gebrechens halbe und, das stethe vast weyet von einander ligen, kan sich kein heer in hysponia lang erhaltten, derhalben man auch in der nacht die stette nicht zuschleusset.

aller Pracht fehlte in den Königsschlössem manches, was uns als unbedingt erforderlich erscheint.... Hier mag nur darauf hingewiesen werden, da $B$ in den französischen Königsschlössem und nicht minder in den spanischen eine uns unbegreifliche Unsauberkeit herrschte, daß die Besucher sich Freiheiten gestatteten, die sich heute einer in dem àrmsten Hause nicht erlauben wūrde. Die Folge davon war, daß bei all dem Luxus die Käume der Palāste von üblen Gerüchen erfüllt erschienen. Die Leute waren aber daran gewöhnt und fanden nichts daran auszusetzen."

1) Man vergleiche mit dieser Schilderung spanischen Herbergswesens im Jahre 1526 das Urteil des Nümbergers Gabriel Tetzel aus den sechziger Jahren des 15 . Jahrhunderts bei Rozmital a. a. O. S. 170 und besonders den beweglichen SchluB seiner Klage: .... also das ich mein, das die Zigeuner in allen landen gar vil herrlicher gehalten werden, dann wir in dem land gehalten wurden. Man findet gar selten huner, ayr, milch, käs noch schmaln, wann es hat kein ku, und ißt selten fleisch, und iBt nichts dann der frucht." 
5 meylen.

Bay onia.

Aldo habe wir das kochgeschir musse umb zwue Cronen geben, welches wir umb 13 Cronen nicht gekaufft hatten[!], und sein ain tag still gelegen.

\section{Castonia.}

4 meil.

Sanct-Vintzentz.

Alhie sein wir ain und dreissig meylen uber die Castanische grosse hayde getzogen, welche eben, sandig und unfruchbar ist, hat das euser merhe nune auff der lencken hant paB gegen Burdeos flyessen, uber welches kain erdreich wonhafftig gegen mitternacht weytter befunden wirt, ') an welches mer wir offte sein gewesen und etzliche meylen daran gerithen.

3 meil.

Ma yestke. ${ }^{2}$ )

Ist ein dorff der Castanischen hayde, darinne wenig weinwachs und vil hirsche befunden.

6 meyl.

Alharre.

Ist ein dorff, darinne man in kainem geflochtten korbe die bynen auffyng.

4 meyl.

Reboffier.

Ist ein klaynes stetlein, darbey der Bastel Barbirer auff der postht sein messer hat verloren.

4 meyl. Moret. ${ }^{8}$ )

Ist ein klaines dorff, hat auch vill hirsche.

1) Eine recht merkwürdige Notiz in Anbetracht der groBen Entdeckungen der Spanier während der letzten Jahrzehnte; ich mōchte sie dahin deuten, daB unser Verfasser nư von Insein im fernen Weltmeer gewuBt hat, wie auch aus folgender Stelle seiner im Jahre 1554 in Basel erschienenen epistolae medicinales (S. 253) hervorgeht: "Nec sua laude fraudandi sunt illustris Ferhnandus[!] Castiliae, ac Iohannes et Henricus ac Emanuel inclyti Portugalliae reges, quorum opera et expensis saluberrimum illud lignum Guaiacum, quod nuper ab Australis Oceani insulis nobis allatum est: quo uno plus commodi miseris mortalibus attulerunt, quam omnes illi avariciae cuniculi, metallorum argenti et auri fossores: qui dum in viscera terrae omnium parentis saeviunt, plus expendunt et insumunt, quam eruunt;“ eine Bemerkung, die auf die Weltanschauung unseres Verfassers ein äußerst interessantes Licht wirft.

2) Magescq. $\quad$ 9) Muret.

Archiv für Kulturgeschichte. V. 

4 meyl.
Bargk. $\left.{ }^{1}\right)$

Ein dorff, von welchem der Bock einen botten geschickt hat nach seinem Rapier zu Moreth verlosen.

\section{Xanthonia. $\left.{ }^{9}\right)$}

6 meyl.

Burdeos.

Aldo endet sich die Castanier grosse hayde, in welcher gantz vil hirsche und flachs gebauet wirt. Alhie sein [wir] wider auff die. alten und ersten strosse komen. Piß gegen Ponth, aldo sein wir wider von der ersten strosse gewichen gegen Econio.

5 meyl.

Econyo.

Ist ein dorff nit weyt von Coniak, welchs auff der rechten hant leut, gehört zu dem hertzogthumb Angulem.

Auff disem wege 3 meylen von Econio sein wir uber das wasser Scherranda ${ }^{\text {g) }}$ gefaren.
4 meil.
One.

Ist ein klaines Stetlein des Hertzogthumbs Angulem.

5 meil. Santh Ligir de Mell.

Ist ein dorff, ein halbe Meyle von der Stat Mell ${ }^{4}$ ) gelegen uff der rechtten hant.

7 meyl.

\section{Lusmer.}

Alhie hat der Ambtman uns wollen auffhalden und nicht lassen passiren, auffs letzte doch uns vergonnet, doch also das wir an kōnigklichem hoffe angetzaigten, das wir bey uns hethen Steffan, des von Rogendorff kayserlichs haubtmans diener, welcher, so wir yn haben am hoffe angetzaigt, hat mussen widerumb in Hyspanien reutten.

Alhie sein wir wider auff die alden strosse komen bis gegen Porthpil, ${ }^{5}$ ) do sich endet Xanthonia und fenget an Thurenia, des Bischoffs von Thors landt.

1) Le Barp. 2) Saintonge. 3) Charente. Melle sur Béronne. 3) Le port de Piles an der Creuse. 


\section{Theurenia das Landt.}

3 meil. Santh-Moer. ${ }^{1}$ )

Ist ein kleynes Stetlein, hatt aber vil volcks.

4 meil.

Mambason. ${ }^{2}$ )

Ist ein kleines Stetlein an einem grossen wasser Scheer ${ }^{3}$ ) gelegen, hatt auff dem berge ein Sloß.

3 meil.

Thors.

Ist ein wollgebauthe stat, darin sant Martin begraben leudt hinder dem hohen altar, welches grab vor etzlichen Jaren mit silber woll gezirt, sey[t]her ietz durch den konig in disen kriegsleuffen seer entplost. In diser stat hatt man allerlay und vil hanthwergksleudt, darin man auch allerley seyden gewant machet. An diser Statt fleuB[t] auch das namhafftige wasser Ligeris genant, frantzosischs Loer.

7 meil.

A m b a B. $)$

Diße Siben meylen sein wir stets an dem uber ${ }^{5}$ ) des wassers Ligiris getzogen, und uff der rechtten handt an den bergen, in welchen uber 150 heuser gehauen sindt, und under der strosse seindt auch heuser, daruber man reyt und feret. Einwoner diser heuser machen seher viel ziegel zu decken und zu pflastern.

Alhie zu AmbaB hat man dem Marschalck in dreyen truchen uberantwurt die silber und ubergolthe Credents, welche der konig zu Franckreich meinem G. $H$. geschenckt hat, welche umb 5000 fl. geschatzt. ${ }^{6}$ )

Alhie sein wieder die alde und erste strosse getzogen biB gen Metz.

Auff disem wege von Pariß bis gein Metz hat es fast in vil dorffern angefangen $\mathrm{zu}$ sterben und sunderlich $\mathrm{zu}$ Sannt

1) Saint-Maure.

9) Montbazon sur l'Indre.

7) Cher.

4) Amboise.

5) Ufer.

6) Vgl. Leodius S. 113a: n[Fridericus] Ambosiam contendit, ubi honorifice a Rege exceptus et aliquot dies contentus et donatus est omni mensae Regiae suppellectili deaurata, valoris sex millium coronatorum." Am 10. August hatte Pfalzgraf Friedrich den franzōsischen Hof in Amboise verlassen. Vgl. Sekretăr Rosso an den Rat der Zehn in Venedig, 10. August 1526: "In questa mattina è partito de qui per Almagna il Conte Palatino venuto di Spagna, apresentato da questa Maestà; e va mal contento di Cesareu. (I Diarii di Marino Sanuto. Venedig 1895. Bd. XLII, Sp. 437.) 
Mihi, da worn die Burger herauß in die welde gewichen, doch haben aldo muessen vor der Stat zu Mittage essen, und dweil wir assen, ist ein gantz hauffe ...1) uff den einen Esel gefallen, welchen wir haben an allen schaden herfurgebrocht.

Frantzosische herberge.

In diesen herbergen ist yderman wolgewart von knechten und megden, und mit woll gekochtter speyß und wilproth, auff welche zeyt er das begert, auch mit sunderlichen raynen bethgewanthte woll versorget, und mit gutter stallunge und aller notturfft des futters.

Iten in Franckreich hats vil Bisthumb, welche doch kaine weltliche gebiet oder obriklieyt haben, und vast seer ungelärthe briester haben, und vast ihre kirchen mit wenig pilden getzirt, und halden alle mit zynen kelchen messe.

Auch wirt grosse Justicia darinne gehalden, also wan das Perlamenth den geweldigisten hertzog auß Franckreich zitirethe, zu Marmelsteyn zu erscheynen erfordert, wen er auff den bestympten Tag nicht erschyne, so hatt er leyb und gut verloren. Auch ist grosse gehorsam und underthenigkheit des volcks, und alles Bauerßvolck thar sich nicht anders dan in grav farbe oder lichtbloe farbe gemaynes grobes Tuchs beklayden.

5 meyl. Santh Trefoer.

Ist ein kleynes Stetlein des Bischofis von Metz, und ist des hertzogen von Lothringen.

5 meil.

Sarburg. ${ }^{2}$ )

Ist ein kleine stath, darinne der graffe von Nassau ${ }^{3}$ ) hoffhaltet, an einem wasser gelegen, durch welchs, so wir rytthen ist der Locay mit sampt dem pferde dareyn gefallen.

3 meil. Lautenbbach.

Ist ein groß dorff Herzog Ludwigs von Grauens zu felde, ${ }^{4}$ ) welchs paur des pfaltzgraven Ludwigs Churfürsten gefangen seint.

1) Lücke im Text.

2) Wie sich aus der ganzen Reiseroute ergibt, ist Saarbrücken gemeint.

8) Johann Ludwig von Nassau-Saarbrücken.

1) Ludwig II. von Pfalz-Zweibrücken-Veldenz. Oeb. 1502, regiert von 1514-1532, Vater des Herzogs Wolfgang von Pfalz-Zweibrücken. 
3 meil.

Landstal.

Ein kleines Stetlein des Frantzen von Sickingen gewest, welches er selbs hat außgebrant, do er von den fursten überzogen wardt.

Alhie sein wir wider uff unser alde strasse biß gein Haydelbergk getzogen, do wir dan wider zu unserm gnedigen herren uff der hirschbrumpft zu Gerberßheym am Rhein komen seyn.

\section{Addicciones.}

Item nach Santh Johanns Baptiste Feyer sein zu Granathen in der nacht zwen erbiden gewest, das sich alle heuser vast seer erschutlten.

Item alle witbfrauen nach irer emenner todte oder geschwister, Bruder und eldern Tode bedecken daz grabe etzliche tage mit einem thebiche, und so sie in die kirche komen, stellen sie ain brynneth liecht auff das grab und ein preth mit einem weyssen tuechlen bedeckh, und knyen darbey, piß das der Briester die messe vollendet hat, darnach get der briester zu dem grabe und bethet Miserere mei deus ader de Profundis, und sprenget das grab mit geweihttem wasser und gibt der frauen seyne hendt ader Casel zu kusen und nymbt das broeth von der Seel wegen; der gebrauch ist in ganlz Hyspanien und in Frankreich. Bey

\section{Ba y o na,}

Item in der Pyschkaya und zu Granatha, auch in Navare in etzlichen stetthen, so man des verstorben Corper zu der erden bestath, so seynd alde weyber bestelt, die zuvor wol gezecht und gespeyst den Toden mit hesslichem geschray beweynen.

6 Meyl. Haydelbergk.

Aldo der Churfurste befestiget sein schloß mit einer zwivechtigen Mauren und Thurmen, welcher ein itzliche funff und zwaintz[ig] schuen lang von grossen werckstücken gemacht, und zwuschen den bayden Mauren ein schutthe 55 schueche breyt; hinder diser schueth und Mauer ist ein tieffer und praytter wassergraben und darnach ein streydende und umblauffende- 
werhe, 12 schue dicke. Aldo seinth wir 14 tage stille gelegen in der hirschbrunfft und seind auff den ersten Tag des monats Octobris weggerithen.

3 meyln.

$$
\text { Hayschbach. }{ }^{1} \text { ) }
$$

Ist ein klaines Stetlein des Pfaltzgraven, daryn wir in der kellereyn sein beherbergt worden.

3 mayllen.

Helbron.

Ist ein reichstat in der große vast Amberg gleichmessig an dem Necker gelegen, und hat der Rath meinem gnedigen Herrn mit dem habern weyn und fischhen verert.

Item ein klain viertl weges hinder diser Stat fenget sich an der Weinßperger tall, desgleichen mit weinwachs Holtz wasser und getrayde in kainem Lande ich gesehen habe, darinne ligdt das Stetlein WeinBpergk mit sambt einem schlosse auff einem weynberge hart daran gelegen, welchs die pauern haben außgeprant und den graven mit sambt ander 17 edelleuten in gegenwart seiner frauen und zwayer Junger Kinder durch die Spisse geiaget ${ }^{2}$ ) und daz aine kneblein $z u$ gedechtnus der zerschnitten hosen auch über die payn und Arme geschnitten und einen edelman oben von dem thurmb herab geworffen, derhalben der bunt und pfaltzgrave diB stetlein haben glat ausgeprant, ${ }^{3}$ ) welchs die pauern widerumb anfangen $z u$ pauen.

3 meyllen.

Oryngen.

Ist ein stat der graffen von Holoch, welche unsern wirth umb 6 hundert gulden straffen, darumb das er den pauern ist auch anhengigk gewest.

1) Wahrscheinlich ist Hilsbach im Kreichgau gemeint.

9) Vgl. Jakob Sturm an den Rat von Straßburg, 22. April 1525 : $\ldots$. . und winsperg schloB und Stat mitt dem Sturm erobert uff den ostertag (16. April), dorin graue Ludwig von Helffenstein sampt sibenzehn vom Adel und ettlich geraysigen zum theyl an der weer ervurgt, zum theyl und namlich den grauen durch die spieß gejagt . ." (Virck: Polit. Corr. von StraBburg I, 196).

3) Vgl. Truchseb Oeorg an Markgraf Kasimir, Neckargartach 22. Mai 1525: Hat ... insbesondere "Winsperg samt einigen dazu gehōrigen Dörfern ihrer mōrderischen, bösen Tat nach geplūndert und ganz ausgebrannt" (Fr. L. Baumann: Akten z. Oesch. d. deutschen Bauernkrieges aus Oberschwaben. Freiburg i. Br. 1877. S. 292 f.). 
Die tagebuchartigen Aufzeichnungen des Dr. Johannes Lange. 439

3 meylen. Halle.

Dyße reichstat leydt an der Tauber in einem grosse gepirge, an welchem wein wechst, hat eine enge Montze und eine [!] enges verschlossens Land, ist halbs an den bergk und halbs in grundt gebauet, in welchem ist ein seichter und praytter Saltzbrun, der do hundert und sybentzig pfannen benuget wassers zu dem saltzsyden, also doch das alleine das halb thayle dyBer pfannen ein woche umb die ander gebraucht werde.

4 meilen. Olewangk. ${ }^{1}$ )

Ist hertzog Henrichs Pfalzgraven bey Rhein Hertzogen In Baiern etc., hat ein vast woll erpauet $\left.{ }^{2}\right)$ schloB.

Hier brechen die Aufzeichnungen ganz unvermittelt ab. Man wird annehmen dürfen, daß die Reisenden von Ellwangen ab dieselbe Route wie auf der Hinreise eingehalten haben.

1) Ellwangen.

2) Bei Jakob Wille: Die deutschen Pfälzer Handschriften, Heidelberg 1903, II, 17 liest man irrtümlich nerspauet". 